\begin{tabular}{|c|l|}
\hline Title & Structure and decay of the pygmy dipole resonance in Ne 26 \\
\hline Author(s) & Kimura, M. \\
\hline Citation & $\begin{array}{l}\text { Physical review C, 95/3), 034331 } \\
\text { https://doi.org/10.1103/PhysRevC.95.034331 }\end{array}$ \\
\hline Issue Date & 2017-03-31 \\
\hline Doc URL & http://hdl.handle.net/2115/65724 \\
\hline Rights & @2017 A merican Physical Society \\
\hline Type & article \\
\hline File Information & PhysRevC95 34331.pdf \\
\hline
\end{tabular}

Instructions for use 


\title{
Structure and decay of the pygmy dipole resonance in ${ }^{26} \mathrm{Ne}$
}

\author{
M. Kimura* \\ Department of Physics, Hokkaido University, Sapporo 060-0810, Japan \\ and Nuclear Reaction Data Centre, Faculty of Science, Hokkaido University, Sapporo 060-0810, Japan \\ (Received 27 November 2016; revised manuscript received 4 February 2017; published 31 March 2017)
}

\begin{abstract}
The low-lying spectra of ${ }^{24,25,26} \mathrm{Ne}$ and the structure of the pygmy dipole resonance (PDR) in ${ }^{26} \mathrm{Ne}$ have been theoretically studied by the antisymmetrized molecular dynamics (AMD) and its extended version called shifted-basis AMD. The calculated energy and strength of the PDR reasonably agree with the observation, and the analysis of the wave function shows that the PDR is dominated by neutron excitation coupled to the quadrupole excited core nucleus ${ }^{25} \mathrm{Ne}$, which explains the observed unexpected decay of PDR to the excited states of ${ }^{25} \mathrm{Ne}$. The large isoscalar component of PDR is also shown and the enhancement of the core excitation in neutron-rich $\mathrm{Ne}$ isotopes is conjectured.
\end{abstract}

DOI: 10.1103/PhysRevC.95.034331

\section{INTRODUCTION}

The low-energy electric dipole $(E 1)$ excitation which emerges well below the giant dipole resonance (GDR) is called pygmy dipole resonance (PDR), and has attracted much interest in this decade [1,2]. It was expected that PDR could be a signature of a novel type of excitation mode peculiar to unstable nuclei, in which the tightly bound inert core oscillates against the surrounding neutron skin [3-5]. Hence, the relationship between the strength of PDR and the growth of neutron skin in many isotope chains was discussed by many authors [6-10]. In addition to this, the PDR is expected to have a strong impact on astrophysical phenomena such as the rapid neutron capture process, and constrains the equation of state of the neutron star matter [7-9,11-13].

Among many observed PDR, that of ${ }^{26} \mathrm{Ne}$ is the one most intensively studied in detail. The experiment performed at RIKEN reported the PDR of ${ }^{26} \mathrm{Ne}$ around $E_{x}=9 \mathrm{MeV}$ with the integrated $E 1$ strength of $B(E 1)=0.49 \pm 0.16 \mathrm{e}^{2} \mathrm{fm}^{2}$ which exhausts approximately $5 \%$ of the Thomas-ReicheKuhn (TRK) sum rule [14]. Many theoretical studies based on the quasiparticle random phase approximation (QRPA) have been performed and have successfully described these observed properties, although the results range between $E_{x}=$ 6-10 MeV and 5\%-10\% of the TRK sum rule depending on the effective interactions used in the calculations [10,15-23]. At the same time, several QRPA calculations pointed out that the PDR of ${ }^{26} \mathrm{Ne}$ is less collective and dominated by a limited number of neutron $1 p 1 h$ excitations. For example, in Refs. $[18,20]$, it was shown that the PDR is dominated by the $v\left(1 s_{1 / 2}^{-1} 1 p_{3 / 2}\right)$ and $v\left(1 s_{1 / 2}^{-1} 1 p_{1 / 2}\right)$ configurations. However, at a glance, these $1 p 1 h$ configurations look contrary to the observed decay pattern of PDR. The dominance of the $1 p 1 h$ configuration such as $v\left(1 s_{1 / 2}^{-1} 1 p_{3 / 2}\right)$ implies that the PDR primary decays to the ground state of ${ }^{25} \mathrm{Ne}$ which has the $v\left(1 s_{1 / 2}^{-1}\right)$ configuration relative to the ground state of ${ }^{26} \mathrm{Ne}$. On the other hand, experimentally, it was found that the PDR of ${ }^{26} \mathrm{Ne}$ predominantly decays into the excited states

"masaaki@ nucl.sci.hokudai.ac.jp of ${ }^{25} \mathrm{Ne}$, not to the ground state [14]. This puzzling situation is casting a question on the structure of ${ }^{26} \mathrm{Ne}$ PDR. Is it possible to understand the structure and decay pattern of ${ }^{26} \mathrm{Ne}$ PDR consistently?

A possible solution for this puzzle is to explicitly include the core excitation to the PDR. If the PDR is dominated by the neutron excitation coupled to the excited ${ }^{25} \mathrm{Ne}$, the observed decay pattern can be straightforwardly understood. In particular, the coupling of the neutron excitation with the low-lying collective modes such as rotation and vibration [24] may play an important role, because it is well known that the neutron excitation across the $N=20$ shell gap induces strong deformation of $\mathrm{Ne}$ isotopes in the island of inversion [25]. Theoretically, the microscopic description of the rotation and vibration coupling requires treatment beyond the linear response. For this purpose, I use antisymmetrized molecular dynamics (AMD) [26,27] and its extended version called shifted-basis AMD [28-31]. In this framework, by the angular momentum projection, the rotational motion is properly described. And, by introducing the basis wave functions in which the centroids of the Gaussian wave packets describing nucleons are "shifted," one is able to describe various particle-hole configurations. This framework was applied to the isoscalar monopole and dipole responses of light stable nuclei $[28,29,31]$ and electric and isoscalar dipole responses of neutron-rich Be isotopes [30].

In this study, the shifted-basis AMD is applied to the electric dipole response of ${ }^{26} \mathrm{Ne}$. It is shown that the observed energy and strength of ${ }^{26} \mathrm{Ne}$ PDR is successfully described by shiftedbasis AMD. Furthermore, it is found that the PDR is dominated by the neutron excitation coupled to the quadrupole excitation of the core, which qualitatively explains the observed decay pattern of PDR. It is also discussed that the PDR has a large isoscalar component at the same time, because of the core excitation.

This paper is organized as follows. The theoretical framework of shifted-basis AMD is explained in Sec. II, and the numerical results for the low-lying spectrum of ${ }^{24,25,26} \mathrm{Ne}$ and the electric dipole response of ${ }^{26} \mathrm{Ne}$ are presented in Sec. III. The analysis of the numerical results is discussed in Sec. IV. I first discuss the splitting of GDR. Then the structure of PDR 
and its isoscalar component are discussed. The final section summarizes this study.

\section{THEORETICAL FRAMEWORK}

Here, I briefly explain the theoretical framework of AMD and the method to extract the single-particle energies and orbits. Then, the generator coordinate method (GCM) and shifted-basis AMD are introduced, which are used to describe the low-lying spectrum and the highly excited $1^{-}$states of ${ }^{26} \mathrm{Ne}$. Using thus-obtained GCM wave functions for the ground and $1^{-}$states, the electric dipole transition strength, response function, and spectroscopic factor are calculated.

\section{A. Antisymmetrized molecular dynamics}

In the AMD framework, one uses the microscopic $A$-body Hamiltonian given as

$$
H=\sum_{i=1}^{A} t(i)+\sum_{i<j}^{A} v_{n}(i j)+\sum_{i<j}^{Z} v_{C}(i j)-t_{\mathrm{c} . \mathrm{m} .} .
$$

In this study, I employ the Gogny D1S interaction [32] as an effective nucleon-nucleon interaction $v_{n}$ and the Coulomb interaction $v_{C}$ is approximated by a sum of seven Gaussians. The center-of-mass kinetic energy $t_{\text {c.m. }}$ is exactly removed, which is essentially important to remove the spurious modes from the isoscalar dipole response.

The intrinsic wave function $\Phi_{\text {int }}$ is represented by a Slater determinant of single-particle wave packets. It is projected to the eigenstate of parity before the variation (parity projection before variation),

$$
\begin{aligned}
& \Phi_{\text {int }}=\mathcal{A}\left\{\varphi_{1}, \varphi_{2}, \ldots, \varphi_{A}\right\}, \\
& \Phi_{\text {int }}^{\pi}=\frac{1+\pi \hat{P}_{x}}{2} \Phi_{\text {int }}, \quad \pi= \pm .
\end{aligned}
$$

Here $\varphi_{i}$ is the single nucleon wave packet having deformed Gaussian form $[33,34]$,

$$
\varphi_{i}(\mathbf{r})=\prod_{\sigma=x, y, z}\left(\frac{2 v_{\sigma}}{\pi}\right)^{\frac{1}{4}} e^{-v_{\sigma}\left(r_{\sigma}-\frac{z_{i \sigma}}{\sqrt{\nu_{\sigma}}}\right)^{2}+\frac{1}{2} Z_{\sigma}} \chi_{i} \xi_{i},
$$

where $\chi_{i}$ is the spinor and $\xi_{i}$ is the isospin fixed to proton or neutron. The $\boldsymbol{Z}_{i}, \boldsymbol{v}$, and $\chi_{i}$ are the parameters of the wave function and determined by the energy variation which minimizes the expectation value of the Hamiltonian,

$$
\widetilde{E}=\frac{\left\langle\Phi^{\pi}|\hat{H}| \Phi^{\pi}\right\rangle}{\left\langle\Phi^{\pi} \mid \Phi^{\pi}\right\rangle}+v_{\beta}(\langle\beta\rangle-\beta)^{2} .
$$

Here the potential $v_{\beta}(\langle\beta\rangle-\beta)^{2}$ imposes the constraint on the quadrupole deformation parameter $\langle\beta\rangle$ defined in Ref. [35]. The magnitude of $v_{\beta}$ is chosen large enough so that $\langle\beta\rangle$ equals to $\beta$ after the energy variation. No constraint was imposed on another quadrupole deformation parameter $\langle\gamma\rangle$, and hence, it always has the optimal value for each $\beta$. As a result of the energy variation, one obtains the optimized wave function denoted by $\Phi_{\text {int }}^{\pi}(\beta)$ for each given value of $\beta$.

\section{B. Single-particle levels}

To investigate the single-particle configuration of the optimized wave functions $\Phi_{\text {int }}^{\pi}(\beta)$, the single-particle Hamiltonian from $\Phi_{\text {int }}\left(\beta_{i}\right)$, is constructed and the neutron single-particle energies and orbits are calculated by diagonalizing it. First, the single-particle wave packets are transformed to the orthonormalized basis,

$$
\tilde{\varphi}_{p}(\boldsymbol{r})=\frac{1}{\sqrt{\lambda_{p}}} \sum_{i=1}^{A} c_{i p} \varphi_{i}(\boldsymbol{r}) .
$$

Here, $\lambda_{p}$ and $c_{i p}$ are the eigenvalues and eigenvectors of the overlap matrix $B_{i j}=\left\langle\varphi_{i} \mid \varphi_{j}\right\rangle$. Using this basis, the singleparticle Hamiltonian is constructed,

$$
\begin{aligned}
h_{p q}= & \left\langle\widetilde{\varphi}_{p}|t| \widetilde{\varphi}_{q}\right\rangle+\sum_{r=1}^{A}\left\langle\widetilde{\varphi}_{p} \widetilde{\varphi}_{r}\left|v_{n}+v_{C}\right| \widetilde{\varphi}_{q} \widetilde{\varphi}_{r}-\widetilde{\varphi}_{r} \widetilde{\varphi}_{q}\right\rangle, \\
& +\frac{1}{2} \sum_{r, s=1}^{A}\left\langle\widetilde{\varphi}_{r} \widetilde{\varphi}_{s}\left|\tilde{\varphi}_{p}^{*} \widetilde{\varphi}_{q} \frac{\delta v_{n}}{\delta \rho}\right| \widetilde{\varphi}_{r} \widetilde{\varphi}_{s}-\widetilde{\varphi}_{s} \widetilde{\varphi}_{r}\right\rangle .
\end{aligned}
$$

The eigenvectors $f_{q \alpha}$ of $h_{p q}$ define the occupied singleparticle orbits $\phi_{\alpha}=\sum_{q=1}^{A} f_{q \alpha} \widetilde{\varphi}_{q}$ and their eigenvalues $\varepsilon_{\alpha}$ are the single-particle energies. To understand the properties of the single-particle orbits, I also calculate the amount of the positive-parity component,

$$
p^{+}=\left|\left\langle\phi_{s}\left|\frac{1+P_{x}}{2}\right| \phi_{s}\right\rangle\right|^{2}
$$

and angular momenta in the intrinsic frame,

$$
\begin{gathered}
j(j+1)=\left\langle\phi_{s}\left|j^{2}\right| \phi_{s}\right\rangle, \quad \Omega=\sqrt{\left\langle\phi_{s}\left|j_{z}^{2}\right| \phi_{s}\right\rangle}, \\
l(l+1)=\left\langle\phi_{s}\left|l^{2}\right| \phi_{s}\right\rangle, \quad m_{l}=\sqrt{\left\langle\phi_{s}\left|l_{z}^{2}\right| \phi_{s}\right\rangle},
\end{gathered}
$$

which corresponds to the asymptotic quantum number of the Nilsson orbits.

\section{Generator coordinate method and shifted-basis AMD}

To describe the ground and excited states, I perform the angular momentum projection and GCM. I also explain the shifted-basis AMD [29-31,36] which is used to generate additional basis wave functions for GCM. First, the eigenstate of the total angular momentum $J$ is projected out from the optimized wave functions $\Phi_{\text {int }}^{\pi}(\beta)$,

$$
\Phi_{K}^{J^{\pi} M}(\beta)=\frac{2 J+1}{8 \pi^{2}} \int d \Omega D_{M K}^{J *}(\Omega) R(\Omega) \Phi_{\text {int }}^{\pi}(\beta) .
$$

Here, $D_{M K}^{J}(\Omega)$ is the Wigner $D$ function and $R(\Omega)$ is the rotation operator. The integrals over three Euler angles $\Omega$ are evaluated numerically. This projected wave function $\Phi_{K}^{J^{\pi} M}(\beta)$ is used as the basis wave functions of GCM.

Then, the wave functions having different quadrupole deformation $\beta$ and projection of angular momentum $K$ are superposed (GCM),

$$
\Psi_{n}^{J^{\pi} M}=\sum_{K=-J}^{J} \sum_{i=1}^{N} e_{K i n} \Phi_{K}^{J^{\pi} M}\left(\beta_{i}\right),
$$


where $N$ is a number of basis wave functions prepared by the energy variation. The coefficients $e_{K i n}$ and eigenenergies $E_{n}^{J^{\pi}}$ are obtained by solving the Hill-Wheeler equation $[37,38]$,

$$
\begin{aligned}
\sum_{K^{\prime} i^{\prime}} H_{K i K^{\prime} i^{\prime}}^{J^{\pi}} e_{K^{\prime} i^{\prime} n} & =E_{n}^{J^{\pi}} \sum_{K^{\prime} i^{\prime}} N_{K i K^{\prime} i^{\prime}}^{J^{\pi}} e_{K^{\prime} i^{\prime} n}, \\
H_{K i K^{\prime} i^{\prime}}^{J^{\pi}} & =\left\langle\Phi_{K}^{J^{\pi} M}\left(\beta_{i}\right)|H| \Phi_{K^{\prime}}^{J^{\pi} M}\left(\beta_{i^{\prime}}\right)\right\rangle, \\
N_{K i K^{\prime} i^{\prime}}^{J^{\pi}} & =\left\langle\Phi_{K}^{J^{\pi} M}\left(\beta_{i}\right) \mid \Phi_{K^{\prime}}^{J^{\pi} M}\left(\beta_{i^{\prime}}\right)\right\rangle .
\end{aligned}
$$

As explained in the next section, the basis wave functions $\Phi_{K}^{J^{\pi} M}\left(\beta_{i}\right)$ are not sufficient to describe GDR, because many of the $1 p 1 h$ configurations which coherently contribute to GDR are missing. To introduce various $1 p 1 h$ configurations, I use the shifted-basis AMD which generates additional basis wave functions as explained below. I denote by $X^{i}$ a set of parameters of the optimized wave function $\Phi_{\text {int }}^{\pi}\left(\beta_{i}\right)$,

$$
X^{i}=\left\{\boldsymbol{Z}_{1}, \ldots, \boldsymbol{Z}_{A}, \boldsymbol{v}, \chi_{1}, \ldots, \chi_{A}\right\},
$$

and introduce new sets of parameters,

$$
\begin{aligned}
X_{j}^{i} & =\left\{\boldsymbol{Z}_{1}^{\prime}, \ldots, \boldsymbol{Z}_{j}^{\prime}, \ldots, \boldsymbol{Z}_{A}^{\prime}, \boldsymbol{v}, \chi_{1}, \ldots, \chi_{A}\right\}, \\
\bar{X}_{j}^{i} & =\left\{\boldsymbol{Z}_{1}^{\prime}, \ldots, \boldsymbol{Z}_{j}^{\prime}, \ldots, \boldsymbol{Z}_{A}^{\prime}, \boldsymbol{v}, \chi_{1}, \ldots, \bar{\chi}_{j}, \ldots, \chi_{A}\right\}, \\
j & =1, \ldots, A,
\end{aligned}
$$

where $\bar{\chi}_{j}$ is the time reversal of $\chi_{j}$, and $\boldsymbol{Z}^{\prime}$ is generated by shifting the original position of the $j$ th Gaussian centroid by $\epsilon \boldsymbol{e}_{\sigma}$

$$
\begin{aligned}
\boldsymbol{Z}_{p}^{\prime} & =\left\{\begin{array}{ll}
\left(\boldsymbol{Z}_{p}+\epsilon \boldsymbol{e}_{\sigma}\right)-\epsilon \boldsymbol{e}_{\sigma} / A, & p=j \\
\boldsymbol{Z}_{p}-\epsilon \boldsymbol{e}_{\sigma} / A, & p \neq j
\end{array},\right. \\
\sigma & =x, y, z .
\end{aligned}
$$

Here, $\boldsymbol{e}_{\sigma}$ are the unit vectors in $x, y$, and $z$ directions, and $\epsilon$ represents the magnitude of the shift which is typically chosen as $\epsilon=0.3 \mathrm{fm}$ in this study. All Gaussian centroids are simultaneously shifted by $-\epsilon \boldsymbol{e}_{\sigma} / A$ to satisfy the relation $\sum_{p=1}^{A} \boldsymbol{Z}_{p}^{\prime}=\sum_{p=1}^{A} \boldsymbol{Z}_{p}=0$, which is needed to avoid the contamination of the spurious center-of-mass excitation. Those new parameter sets generate $6 N A(2 J+1)$ new wave functions denoted by $\Phi_{K}^{J^{\pi} M}\left(\beta_{i} ; X_{j}^{i}\right)$ and $\Phi_{K}^{J^{\pi}}{ }^{M}\left(\beta_{i} ; \bar{X}_{j}^{i}\right)$ to be used as additional basis wave functions. The meaning of the shift of Gaussian centroids is explained in Appendix B. If one performs GCM with only those new basis functions, the GCM wave function is given as

$$
\Psi_{n}^{J^{\pi} M}=\sum_{K i j}\left(f_{K i j n} \Phi_{K}^{J^{\pi} M}\left(\beta_{i} ; X_{j}\right)+g_{K i j n} \Phi_{K}^{J^{\pi} M}\left(\beta_{i} ; \bar{X}_{j}\right)\right),
$$

and if I include all basis functions,

$$
\begin{aligned}
\Psi_{n}^{J^{\pi} M}= & \sum_{K i} e_{K i n} \Phi_{K}^{J^{\pi} M}\left(\beta_{i}\right)+\sum_{K i j}\left(f_{K i j n} \Phi_{K}^{J^{\pi} M}\left(\beta_{i} ; X_{j}\right)\right. \\
& \left.+g_{K i j n} \Phi_{K}^{J^{\pi} M}\left(\beta_{i} ; \bar{X}_{j}\right)\right),
\end{aligned}
$$

where the coefficients of superposition are determined by solving the Hill-Wheeler equation. Hereafter, I denote the GCM calculations using the wave function Eqs. (12), (20), and (21) as $\beta$ GCM, shifted-basis GCM, and full GCM, respectively.

\section{Dipole transition strength}

Using the GCM wave functions for the ground and excited $1^{-}$states, I calculate the electric dipole transition probability $B(E 1)$ and excitation function $S(E 1 ; E)$ defined as

$$
\begin{aligned}
\mathcal{M}_{\mu}(E 1) & =\frac{N}{A} \sum_{i \in \mathrm{p}} r_{i} Y_{1 \mu}(\hat{r})-\frac{Z}{A} \sum_{i \in \mathrm{n}} r_{i} Y_{1 \mu}(\hat{r}), \\
B\left(E 1 ; 0_{1}^{+} \rightarrow 1_{n}^{-}\right) & =\sum_{\mu}\left|\left\langle\Psi_{n}^{1^{-} \mu}\left|\mathcal{M}_{\mu}(E 1)\right| \Psi_{1}^{0^{+} 0}\right\rangle\right|^{2} \\
S(E 1 ; E) & =\sum_{n} \frac{\Gamma / 2}{\pi} \frac{B\left(E 1 ; 0_{1}^{+} \rightarrow 1_{n}^{-}\right)}{\left(E-E_{n}\right)^{2}+\Gamma^{2} / 4}
\end{aligned}
$$

where the smearing width is chosen as $\Gamma=1 \mathrm{MeV}$. The energy weighted and nonweighted sums,

$$
m_{n}=\int d E B(E 1 ; E) E^{n},
$$

are also evaluated to see the centroid energy of GDR and the convergence of the calculation. In addition to the $E 1$ response, I also calculated the isoscalar dipole transitions whose operator is defined as

$$
\mathcal{M}_{\mu}(I S 1)=\sum_{i=1}^{A}\left(\boldsymbol{r}_{i}-\boldsymbol{r}_{\mathrm{c} . \mathrm{m} .}\right)^{2} \mathcal{Y}_{1 \mu}\left(\boldsymbol{r}_{i}-\boldsymbol{r}_{\mathrm{c} . \mathrm{m} .}\right),
$$

where $\boldsymbol{r}_{\text {c.m. }}$ denotes the center of mass of the system and the solid spherical harmonics is defined as $\mathcal{Y}_{l m}(\boldsymbol{r})=r^{l} Y_{l m}(\hat{r})$. The transition probability $B(I S 1)$ and excitation function $S(I S 1 ; E)$ are defined in the same manner as the $E 1$ transition.

\section{E. Overlap amplitude and spectroscopic factor}

To investigate the structure of the $1^{-}$states, I calculated the overlap amplitude and spectroscopic factor. The overlap amplitude is defined as the overlap between the wave functions of nuclei with mass $A$ and $A+1$. For example, the overlap amplitude for ${ }^{26} \mathrm{Ne}$ is defined as

$$
\varphi(\boldsymbol{r})=\sqrt{A+1}\left\langle\Psi_{n^{\prime}}^{J^{\prime \pi^{\prime}} M^{\prime}}\left({ }^{25} \mathrm{Ne}\right) \mid \Psi_{n}^{J^{\pi} M}\left({ }^{26} \mathrm{Ne}\right)\right\rangle .
$$

If the wave functions for ${ }^{25} \mathrm{Ne}$ and ${ }^{26} \mathrm{Ne}$ are given by $\beta \mathrm{GCM}$, Eq. (27) reads

$$
\begin{aligned}
\varphi(\boldsymbol{r})= & \sqrt{A+1} \sum_{K i K^{\prime} i^{\prime}} e_{K^{\prime} i^{\prime} n^{\prime}}^{*} e_{K i n} \\
& \times\left\langle\Phi_{K^{\prime}}^{J^{\prime \pi^{\prime}} M^{\prime}}\left(\beta_{i^{\prime}} ;{ }^{25} \mathrm{Ne}\right) \mid \Phi_{K}^{J^{\pi} M}\left(\beta_{i} ;{ }^{26} \mathrm{Ne}\right)\right\rangle .
\end{aligned}
$$

Using Eqs. (A8) and (A9), it is calculated as

$$
\begin{aligned}
\varphi(\boldsymbol{r})= & \sum_{j l} C_{J^{\prime} M^{\prime}, j M-M^{\prime}}^{J M} \varphi_{j l}(r)\left[Y_{l}(\hat{r}) \otimes \chi\right]_{j M-M^{\prime}} \\
\varphi_{j l}(r)= & \sum_{K i K^{\prime} i^{\prime}} e_{K^{\prime} i^{\prime} n^{\prime}}^{*} e_{K i n} \sum_{k} C_{J^{\prime} K^{\prime}-k, j k}^{J K} \sum_{p=1}^{26}(-)^{p} \psi_{j l k}^{(p)}(r ; i) \\
& \times \frac{2 J^{\prime}+1}{8 \pi^{2}} \int d \Omega D_{K^{\prime} K-k}^{J^{\prime} *}(\Omega) \operatorname{det} B^{\left(p ; i i^{\prime}\right)}(\Omega)
\end{aligned}
$$



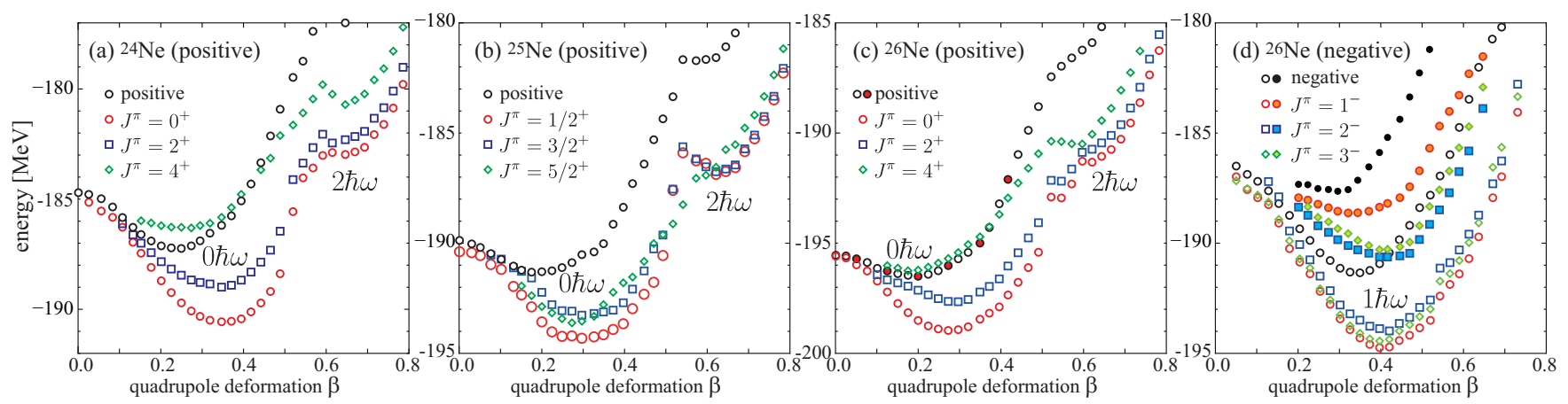

FIG. 1. The energy curves as functions of quadrupole deformation parameter $\beta$ for ${ }^{24} \mathrm{Ne},{ }^{25} \mathrm{Ne}$, and ${ }^{26} \mathrm{Ne}$. Symbols denoted by "positive" or "negative" show the results of the energy variation after the parity projection, while others show those after the angular momentum projection. For the negative-parity states of ${ }^{26} \mathrm{Ne}$, two different single-particle configurations were obtained, which are shown by open and filled symbols.

Once the overlap amplitude is calculated, its integral yields the spectroscopic factor,

$$
S_{j l}=\int_{0}^{\infty} r^{2} d r\left|\varphi_{j l}(r)\right|^{2} .
$$

The details of the above expressions are explained in Appendix A. It is straightforward to derive corresponding expressions for shifted-basis GCM and full GCM wave functions.

\section{RESULTS}

In this section, I first show the low-lying level scheme of ${ }^{24} \mathrm{Ne},{ }^{25} \mathrm{Ne}$, and ${ }^{26} \mathrm{Ne}$ obtained by $\beta$ GCM. Then I compare the electric dipole response functions obtained by $\beta \mathrm{GCM}$, shifted-basis GCM, and full GCM.

\section{A. Results of energy variation and single-particle configurations}

Figure 1 shows the energy curves for positive-parity states of ${ }^{24} \mathrm{Ne}$ and ${ }^{25} \mathrm{Ne}$, and those for positive- and negative-parity states of ${ }^{26} \mathrm{Ne}$ obtained by the energy variation after the parity projection and the angular momentum projection. All nuclei discussed here locate out of the island of inversion, and hence, their ground states are dominated by the $0 \hbar \omega$ (normal) configurations. The strongly deformed $2 \hbar \omega$ (intruder) configurations locate approximately $7 \mathrm{MeV}$ above the normal configurations in all nuclei. After the angular momentum projection, the energy minima of $0^{+}$or $1 / 2^{+}$ states corresponding to the ground states have non-negligible deformations that are $\beta \simeq 0.35$ for ${ }^{24} \mathrm{Ne}$ and $\beta \simeq 0.30$ for ${ }^{25} \mathrm{Ne}$ and ${ }^{26} \mathrm{Ne}$. For the negative-parity states of ${ }^{26} \mathrm{Ne}$, I have obtained two energy minima which have different internal structures.

The single-particle configurations of the positive- and negative-parity minima of ${ }^{26} \mathrm{Ne}$ can be understood from the properties of single-particle orbits listed in Table I. At the energy minimum of the positive-parity state, the most weakly bound two neutrons occupy the $\left[2111 / 2^{+}\right]$Nilsson orbit which originates in the spherical $1 s_{1 / 2}$ orbit [Table I(a)]. Owing to the spherical nature of this orbit, the deformation of neutron distribution is smaller than that of proton distribution as seen in its density profile shown in Fig. 2(a), which reduces the deformation of the system compared to ${ }^{24} \mathrm{Ne}$ as mentioned above.
The $1^{-}$states shown by open circles in Fig. 1(d) have the energy minimum located around $\beta=0.40$ at approximately $4 \mathrm{MeV}$ above the positive-parity minimum, whose singleparticle levels are listed in Table I(b). One sees that the protons do not change their configuration from the positiveparity minimum, but a valence neutron is excited from the [211 $\left.1 / 2^{+}\right]$orbit to the $\left[3301 / 2^{-}\right]$orbit which corresponds

TABLE I. The valence four proton and six neutron orbits of ${ }^{26} \mathrm{Ne}$ at the energy minima of (a) positive parity with $0 \hbar \omega$ configuration, (b) negative parity with neutron excitation, and (c) negative parity with proton excitation. The single-particle energy $\varepsilon$ is given in $\mathrm{MeV}$. Other quantities are defined by Eqs. (8)-(10). The Nilsson asymptotic quantum numbers $\left[N n_{z} m_{l} \Omega^{\pi}\right]$ deduced from those properties are also given.

(a) Positive minimum at $\beta=0.30$

\begin{tabular}{lrcccccc}
\hline Orbit & \multicolumn{1}{c}{$\varepsilon$} & $p^{+}$ & $j$ & $l$ & $m_{l}$ & $\Omega$ & {$\left[N n_{z} m_{l} \Omega^{\pi}\right]$} \\
\hline$\pi 1,2$ & -17.0 & 0.99 & 2.3 & 1.9 & 0.5 & 0.5 & {$\left[2201 / 2^{+}\right]$} \\
$\pi 3,4$ & -24.3 & 0.01 & 0.7 & 1.0 & 1.0 & 0.5 & {$\left[1011 / 2^{-}\right]$} \\
$v 1,2$ & -5.2 & 0.99 & 1.5 & 1.5 & 0.8 & 0.5 & {$\left[2111 / 2^{+}\right]$} \\
$v 3,4$ & -7.3 & 0.99 & 2.5 & 2.0 & 2.0 & 2.5 & {$\left[2025 / 2^{+}\right]$} \\
$v 5,6$ & -10.1 & 0.99 & 2.5 & 2.0 & 1.1 & 1.5 & {$\left[2113 / 2^{+}\right]$}
\end{tabular}

(b) Negative minimum at $\beta=0.40$ (neutron ex.)

\begin{tabular}{lccccccc}
\hline Orbit & \multicolumn{1}{c}{$\varepsilon$} & $p^{+}$ & $j$ & $l$ & $m_{l}$ & $\Omega$ & {$\left[N n_{z} m_{l} \Omega^{\pi}\right]$} \\
\hline$\pi 1,2$ & -18.8 & 0.99 & 2.3 & 1.8 & 0.4 & 0.5 & {$\left[2201 / 2^{+}\right]$} \\
$\pi 3,4$ & -23.1 & 0.10 & 0.7 & 1.1 & 1.0 & 0.5 & {$\left[1011 / 2^{-}\right]$} \\
$v 1$ & -1.4 & 0.00 & 2.9 & 2.5 & 0.4 & 0.5 & {$\left[3301 / 2^{-}\right]$} \\
$v 2$ & -5.2 & 0.99 & 1.7 & 1.7 & 0.9 & 0.5 & {$\left[2111 / 2^{+}\right]$} \\
$v 3,4$ & -6.3 & 0.99 & 2.5 & 2.0 & 2.0 & 2.5 & {$\left[2025 / 2^{+}\right]$} \\
$v 5,6$ & -10.1 & 0.99 & 2.5 & 2.1 & 1.1 & 1.5 & {$\left[2113 / 2^{+}\right]$}
\end{tabular}

(c) Negative minimum at $\beta=0.32$ (proton ex.)

\begin{tabular}{lccccccc}
\hline Orbit & $\varepsilon$ & $p^{+}$ & $j$ & $l$ & $m_{l}$ & $\Omega$ & {$\left[N n_{z} m_{l} \Omega^{\pi}\right]$} \\
\hline$\pi 1,2$ & -16.7 & 0.99 & 2.4 & 1.9 & 0.6 & 0.7 & {$\left[2201 / 2^{+}\right]$} \\
$\pi 3$ & -20.1 & 0.53 & 1.8 & 1.6 & 1.0 & 1.0 & {$\left[2113 / 2^{+}\right]+\left[1011 / 2^{-}\right]$} \\
$\pi 4$ & -25.0 & 0.00 & 0.7 & 1.0 & 1.0 & 0.5 & {$\left[1011 / 2^{-}\right]$} \\
$v 1,2$ & -5.1 & 0.99 & 1.5 & 1.4 & 0.8 & 0.6 & {$\left[2111 / 2^{+}\right]$} \\
$v 3,4$ & -7.2 & 0.99 & 2.5 & 2.0 & 2.0 & 2.5 & {$\left[2025 / 2^{+}\right]$} \\
$v 5,6$ & -10.3 & 0.99 & 2.5 & 2.0 & 1.1 & 1.5 & {$\left[2113 / 2^{+}\right]$} \\
\hline \hline
\end{tabular}




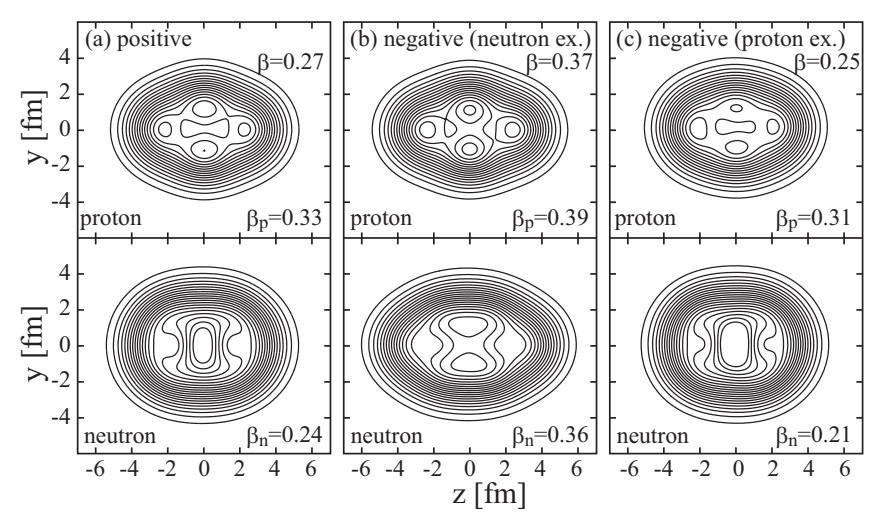

FIG. 2. Intrinsic density distributions of ${ }^{26} \mathrm{Ne}$ at energy minima of positive- and negative-parity states. Upper (lower) panels show proton (neutron) distributions. $\beta, \beta_{p}$, and $\beta_{n}$, respectively, denote the quadrupole deformation of matter (proton+neutron), proton, and neutron density distributions at each minima.

to the neutron excitation from $s d$ to $p f$ shells. The neutron particle and hole enlarge the deformation of the neutron distribution, and as a result, the deformation of the system is much larger than the positive-parity minimum [Fig. 2(b)]. It is noted that the degeneracy of the single-particle orbit is lost in this configuration because the time reversal symmetry is broken. Therefore, the single-particle energies and other properties listed in the table are averaged for the pair of the approximately degenerated orbits. Other $1^{-}$states shown by filled circles in Fig. 1(c) have the minimum approximately $8 \mathrm{MeV}$ above the positive-parity minimum around $\beta=0.32$ whose single-particle configuration is given in Table I(c). In this state, the neutron configuration is unchanged from the positive-parity minimum, but the third proton occupies the orbit which is an admixture of the positive and negative parity. From the properties of this orbit, I deduced that the $\left[\begin{array}{ll}211 & 3 / 2^{+}\end{array}\right]$and $\left[\begin{array}{ll}101 & 1 / 2^{-}\end{array}\right]$orbits are mixed. Therefore, when the intrinsic wave function is projected to the negative parity, this configuration approximately corresponds to the

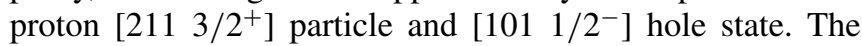
proton particle and hole reduce the deformation of the proton distribution [Fig. 2(c)] leading to the reduction of the total system deformation. The $J^{\pi}=2^{-}$and $3^{-}$states shown by open and filled boxes and diamonds have the same singleparticle configurations as the $J^{\pi}=1^{-}$states mentioned above.

Although I do not show the calculated results, it is noted that the single-particle configurations of ${ }^{24} \mathrm{Ne}$ and ${ }^{25} \mathrm{Ne}$ are understood in the same way. Namely, their ground states are dominated by the normal configuration, while the $2 \hbar \omega$ excited configuration has two neutrons in the $\left[\begin{array}{lll}330 & 1 / 2^{+}\end{array}\right]$ orbit. The negative-parity states of ${ }^{25} \mathrm{Ne}$ also have two energy minima. The lowest minimum has a neutron excitation from the $\left[2111 / 2^{+}\right]$orbit to the $\left[3301 / 2^{+}\right]$orbit, while the upper minimum has a proton excitation from the $\left[1011 / 2^{-}\right]$orbit to the $\left[2113 / 2^{+}\right]$orbit, which is qualitatively the same as ${ }^{26} \mathrm{Ne}$.

The results obtained by the energy variation and angular momentum projection are summarized as follows: $(1)^{25} \mathrm{Ne}$ and ${ }^{26} \mathrm{Ne}$ have positive-parity minimum with smaller deformation compared to that of ${ }^{24} \mathrm{Ne}$. This is because of the valence neutrons occupying the $\left[2111 / 2^{+}\right]$orbit which originates in the spherical $1 s_{1 / 2}$ orbit. (2) ${ }^{25} \mathrm{Ne}$ and ${ }^{26} \mathrm{Ne}$ have two negativeparity minima having different single-particle configurations.

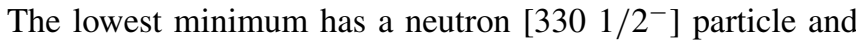
a $\left[2111 / 2^{+}\right]$hole. The neutron particle and hole enlarge the deformation compared to the positive-parity minimum. (3) Another minimum has a proton $\left[2113 / 2^{+}\right]$particle and $\left[1011 / 2^{-}\right]$hole, which reduces the nuclear deformation.

\section{B. Low-lying energy spectra obtained by $\boldsymbol{\beta}$ GCM}

The energy spectra of ${ }^{24} \mathrm{Ne},{ }^{25} \mathrm{Ne}$, and ${ }^{26} \mathrm{Ne}$ obtained by $\beta$ GCM are shown in Fig. 3. I first examine the result for ${ }^{24} \mathrm{Ne}$. For this nucleus, I have not obtained any negative-parity states below $5 \mathrm{MeV}$. The ground band members $\left(0^{+}, 2^{+}\right.$, and $4^{+}$ states) having normal configurations are reasonably described showing a vibrational spectrum, although the excitation energies of the first $2^{+}$and $4^{+}$states are slightly underestimated. The present result also gives the reasonable $B\left(E 2 ; 0^{+} \rightarrow 2^{+}\right)$ value which is consistent with the observed value as listed in Table II. The second $2_{2}^{+}$state is followed by the $3^{+}$state at approximately $5 \mathrm{MeV}$ which also has normal configuration and constitutes a $\gamma$ vibrational band. Their relatively small excitation energies imply the $\gamma$ softness of this nucleus, although the $3^{+}$state has not been experimentally identified yet. The $0_{2}^{+}$state having the intruder configuration locates at $5.62 \mathrm{MeV}$ which slightly overestimates the observed excitation

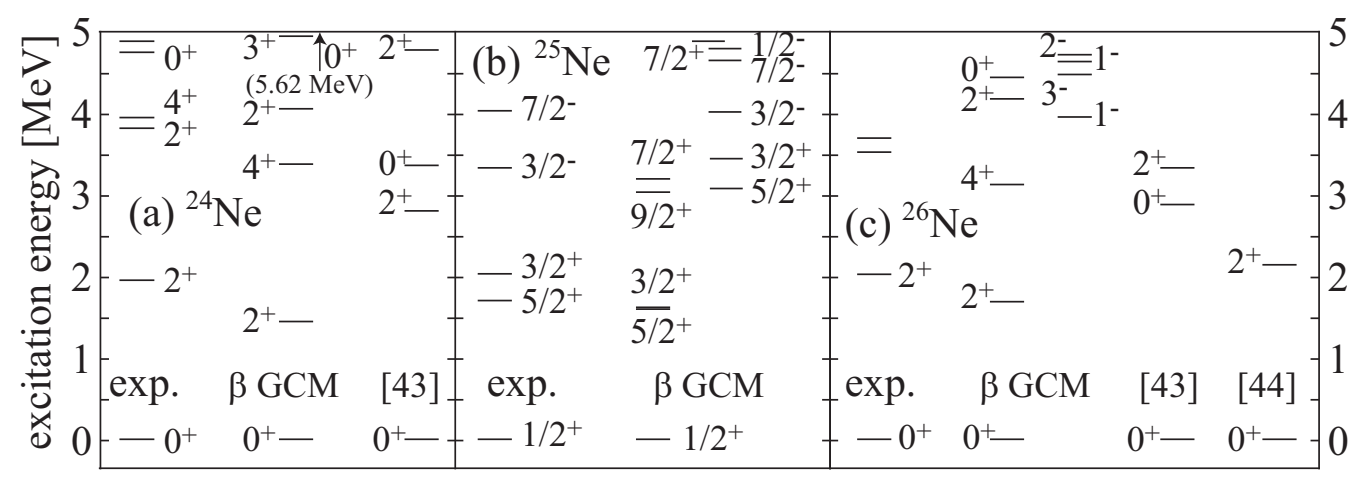

FIG. 3. Observed [39-42] and calculated spectrum of ${ }^{24} \mathrm{Ne},{ }^{25} \mathrm{Ne}$, and ${ }^{26} \mathrm{Ne}$. The results of the HFB-based theoretical calculations [43,44] which also use Gogny D1S interaction are also shown. 
TABLE II. Reduced E2 transition probabilities for low-lying states of neon isotopes in the unit of $e^{2} \mathrm{fm}^{4}$. Numbers in parentheses are the experimental values taken from Refs. $[45,46]$.

\begin{tabular}{|c|c|c|c|c|c|}
\hline \multicolumn{2}{|c|}{${ }^{24} \mathrm{Ne}$} & \multicolumn{2}{|l|}{${ }^{25} \mathrm{Ne}$} & \multicolumn{2}{|c|}{${ }^{26} \mathrm{Ne}$} \\
\hline$J_{i} \rightarrow J_{f}$ & $B(E 2)$ & $J_{i} \rightarrow J_{f}$ & $B(E 2)$ & $J_{i} \rightarrow J_{f}$ & $B(E 2)$ \\
\hline $2_{1}^{+} \rightarrow 0_{1}^{+}$ & $24.3(28)$ & $3 / 2_{1}^{+} \rightarrow 1 / 2_{1}^{+}$ & 35.9 & $2_{1}^{+} \rightarrow 0_{1}^{+}$ & $31.1(28)$ \\
\hline $2_{2}^{+} \rightarrow 0_{1}^{+}$ & 2.3 & $5 / 2_{1}^{+} \rightarrow 1 / 2_{1}^{+}$ & 27.4 & $2_{2}^{+} \rightarrow 0_{1}^{+}$ & 6.7 \\
\hline $4_{1}^{+} \rightarrow 2_{1}^{+}$ & 17.5 & $5 / 2_{1}^{+} \rightarrow 3 / 2_{1}^{+}$ & 1.6 & $4_{1}^{+} \rightarrow 2_{1}^{+}$ & 32.1 \\
\hline $4_{1}^{+} \rightarrow 2_{2}^{+}$ & 1.5 & $7 / 2_{1}^{+} \rightarrow 3 / 2_{1}^{+}$ & 40.9 & $4_{1}^{+} \rightarrow 2_{2}^{+}$ & 2.3 \\
\hline $3_{1}^{+} \rightarrow 2_{1}^{+}$ & 6.5 & $7 / 2_{1}^{+} \rightarrow 5 / 2_{1}^{+}$ & 6.5 & $3_{1}^{+} \rightarrow 2_{1}^{+}$ & 6.9 \\
\hline \multirow[t]{2}{*}{$3_{1}^{+} \rightarrow 2_{2}^{+}$} & 18.8 & $9 / 2_{1}^{+} \rightarrow 5 / 2_{1}^{+}$ & 26.1 & $3_{1}^{+} \rightarrow 2_{2}^{+}$ & 1.3 \\
\hline & & $9 / 2_{1}^{+} \rightarrow 7 / 2_{1}^{+}$ & 1.9 & & \\
\hline
\end{tabular}

energy of $4.77 \mathrm{MeV}$. On the other hand, the Hartree-FockBogoliubov (HFB) calculation with the angular momentum projection and generator coordinate method (AMPGCM) [43] which also uses the Gogny D1S interaction underestimates the $0_{2}^{+}$state energy $(2.9 \mathrm{MeV})$. This difference may be from the difference of the theoretical treatment. In the present calculation, the pairing effect is not explicitly included and AMPGCM calculation assumes the axial symmetry.

The spectrum of ${ }^{25} \mathrm{Ne}$ is shown in Fig. 3(b). In the low-lying positive-parity states, similar to ${ }^{26} \mathrm{Ne}$, the last neutron occupies the $\left[2111 / 2^{+}\right]$orbit which originates in the spherical $1 s_{1 / 2}$ orbit. Therefore, the ground state is the $1 / 2^{+}$state and it is followed by the $3 / 2^{+}$to $9 / 2^{+}$states to constitute the ground band. The energies of the band member states $\left(3 / 2^{+}\right.$and $5 / 2^{+}$ states) reasonably agree with the observation, although the $7 / 2^{+}$and $9 / 2^{+}$states have not been observed yet. This ground band is interpreted as the coupling of the $1 s_{1 / 2}$ neutron to the ground band of ${ }^{24} \mathrm{Ne}$. Namely, ${ }^{24} \mathrm{Ne}\left(2^{+}\right) \otimes 1 s_{1 / 2}$ yields the $5 / 2^{+}-3 / 2^{+}$doublet and ${ }^{24} \mathrm{Ne}\left(4^{+}\right) \otimes 1 s_{1 / 2}$ yields the $9 / 2^{+}$$7 / 2^{+}$doublet. The calculated and observed $B(E 2)$ values and the spectroscopic factors listed in Tables II and III appear to support this interpretation, although the $0_{1}^{+} \otimes d_{3 / 2}$ component is not small for the $3 / 2^{+}$state.

The negative-parity states of ${ }^{25} \mathrm{Ne}$ provide approximate information about the size of the $N=20$ shell gap in this

TABLE III. Single-particle spectroscopic factors for the lowlying states of ${ }^{25} \mathrm{Ne}$ and the ground state of ${ }^{26} \mathrm{Ne}$. Numbers in parentheses are the experimental values taken from Refs. [42,47].

\begin{tabular}{lcccc}
\hline \hline & $0_{1}^{+} \otimes l_{j}$ & $2_{1}^{+} \otimes s_{1 / 2}$ & $2_{1}^{+} \otimes d_{5 / 2}$ & $2_{1}^{+} \otimes d_{3 / 2}$ \\
\hline${ }^{25} \mathrm{Ne}\left(1 / 2_{1}^{+}\right)$ & $0.57(0.80)$ & & 0.52 & 0.14 \\
${ }^{25} \mathrm{Ne}\left(3 / 2_{1}^{+}\right)$ & $0.21(0.44)$ & 0.15 & 0.00 & 0.05 \\
${ }^{25} \mathrm{Ne}\left(5 / 2_{1}^{+}\right)$ & $0.07(0.10)$ & 0.49 & 0.10 & 0.10 \\
& $0_{1}^{+} \otimes l_{j}$ & $2_{1}^{+} \otimes p_{3 / 2}$ & $2_{1}^{+} \otimes f_{7 / 2}$ & \\
${ }^{25} \mathrm{Ne}\left(3 / 2_{1}^{-}\right)$ & $0.40(0.75)$ & 0.26 & 0.08 \\
${ }^{25} \mathrm{Ne}\left(7 / 2_{1}^{-}\right)$ & $0.64(0.73)$ & 0.04 & 0.05 & \\
& $1 / 2_{1}^{+} \otimes s_{1 / 2}$ & $3 / 2_{1}^{+} \otimes d_{3 / 2}$ & $5 / 2_{1}^{+} \otimes d_{5 / 2}$ & \\
& $1.04(1.4)$ & $0.55(0.5)$ & $1.47(1.3)$ & \\
\hline \hline
\end{tabular}

mass region. The calculated $3 / 2^{-}$and $7 / 2^{-}$states locate at 4.0 and $4.7 \mathrm{MeV}$ and slightly overestimate the observed values of 3.3 and $4.0 \mathrm{MeV}[41,42,47-50]$. The observed and calculated spectroscopic factors for those negative-parity states are large, and hence, their excitation energies are good measures for the $p_{3 / 2}$ and $f_{7 / 2}$ single-particle energies. It is interesting to note that the order of the $3 / 2^{-}$and $7 / 2^{-}\left(1 p_{3 / 2}\right.$ and $\left.0 f_{7 / 2}\right)$ are already inverted in this nucleus, which explains the reason of the $p_{3 / 2}$ neutron-halo formation in ${ }^{31} \mathrm{Ne}$ [51-55].

The low-lying spectrum of ${ }^{26} \mathrm{Ne}$ is shown in Fig. 3(c). Experimentally, the first $2^{+}$state is known at $2.0 \mathrm{MeV}$ and two states without definite spin-parity assignment are observed at 3.5 and $3.7 \mathrm{MeV}$. The present calculation yields the first $2^{+}$state at $1.8 \mathrm{MeV}$ and predict the $4^{+}$state at $3.2 \mathrm{MeV}$. Those yrast states constitute the ground band dominated by the $\left[2111 / 2^{+}\right]^{2}$ configuration or by the $\left(1 s_{1 / 2}\right)^{2}$ configuration, which is confirmed from the observed and calculated spectroscopic factors listed in Table III. The calculation also predicts the second $0^{+}$state at $4.5 \mathrm{MeV}$ which is dominated by the intruder $\left[\begin{array}{lll}330 & 1 / 2^{+}\end{array}\right]^{2}$ configuration. Similar to ${ }^{24} \mathrm{Ne}$, this nucleus also has the low-lying $K^{\pi}=2^{+}$band owing to its softness against the $\gamma$ deformation. It is constituted by the second $2^{+}$state at $4.2 \mathrm{MeV}$ and the $3^{+}$state at $5.1 \mathrm{MeV}$. Those nonyrast states may correspond to one of the observed states at 3.5 and 3.7 MeV. The HFB calculations with AMPGCM [43] overestimate the $2_{1}^{+}$state energy by approximately $1.5 \mathrm{MeV}$, while the other HFB-based GCM calculation with the Gaussian overlap approximation [44] reasonably describes the observed energy. Thus, the numerical results are dependent on the theoretical models to some extent.

The low-lying $1^{-}$states of ${ }^{26} \mathrm{Ne}$ are of particular interest because of their relationship to the pygmy dipole resonance. The lowest energy minimum in Fig. 1(c) which is dominated by a neutron excitation yields a group of the negative parity states around 4-5 MeV shown in Fig. 3(c). It generates $1_{1}^{-}$ and $1_{2}^{-}$states at 4.0 and $4.5 \mathrm{MeV}$. The energy minimum with a proton excitation yields another group of $1^{-}$states around 8-11 MeV.

\section{Electric dipole response of ${ }^{26} \mathrm{Ne}$}

Figure 4(a) shows the electric dipole strength functions where the histograms show the results of $\beta$ GCM, shiftedbasis GCM, and fill GCM. The solid line shows the full GCM result smeared with the Lorentzian with $1-\mathrm{MeV}$ width. In the result of $\beta \mathrm{GCM}$ (blue histogram), there are tiny peaks around 5-10 MeV which are the neutron and proton excited states explained in the previous section. On the other hand, there is almost no prominent strength above $10 \mathrm{MeV}$, which means that $\beta$ GCM is insufficient to describe the highly excited $1^{-}$states, in particular, the GDR to which various $1 p 1 h$ configurations coherently contribute.

The shifted-basis GCM [green histogram in Fig. 4(a)] overcomes this problem. It yields two large peaks around 21 and $28 \mathrm{MeV}$ which correspond to the GDR. The origin of this splitting is attributed to the deformation of the ground state and is discussed in the next section. The energy weighted sum $\left(m_{1}\right)$ listed in Table IV is evidently increased compared with $\beta$ GCM. It is consistent with other theoretical calculations 


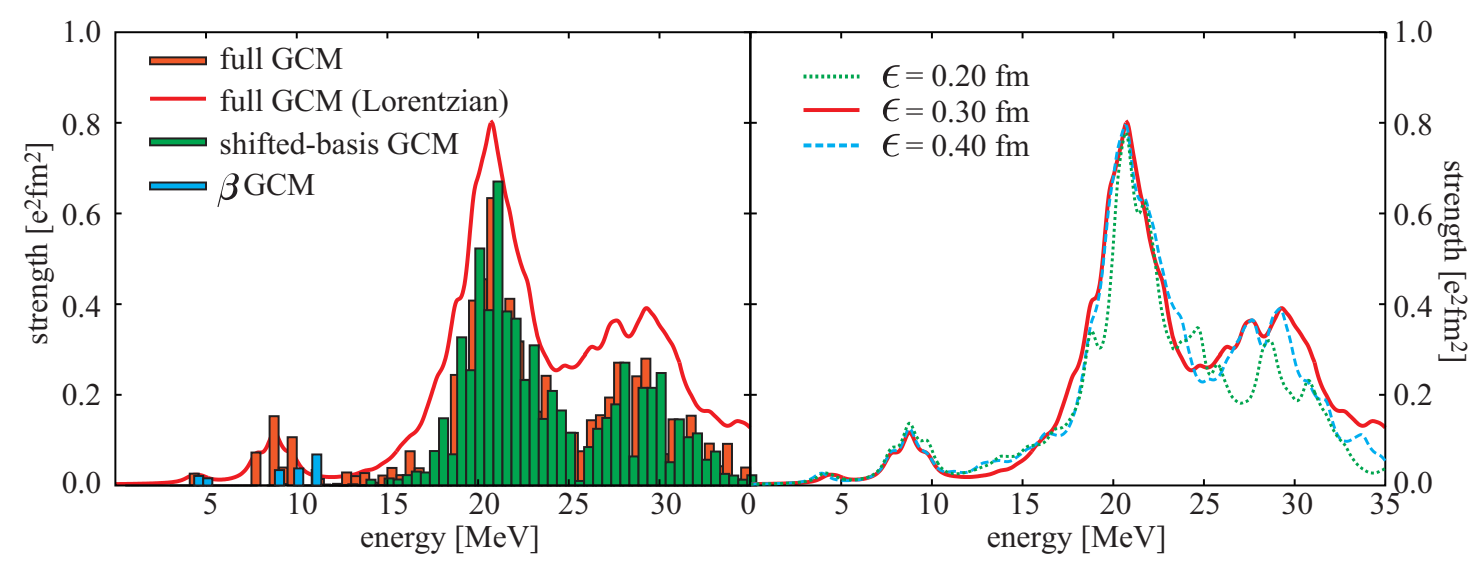

FIG. 4. (a) The electric dipole strength functions of ${ }^{26} \mathrm{Ne}$. The histograms show the results calculated by $\beta$ GCM, shifted-basis GCM and full GCM summed in the energy bin of $0.5 \mathrm{MeV}$ width. The solid line shows the result of full GCM smeared by the Lorentzian with 1-MeV width. (b) Comparison of the results of full GCM calculations in which the magnitude of the shift is changed from 0.20 to $0.40 \mathrm{fm}$.

with Gogny D1S interaction and much larger than the TRK sum rule $\left(92 e^{2} \mathrm{fm}^{2} \mathrm{MeV}\right)$ because of the momentum and isospin dependence of the Gogny D1S. Thus, the shifted-basis GCM successfully describes GDR by introducing various $1 p 1 h$ configurations using the shifted Gaussian wave packets. However, the tiny peaks around $5-10 \mathrm{MeV}$ are not clear in the shifted-basis GCM compared to the $\beta$ GCM. This may mean that the single-particle wave functions such as $\left[3301 / 2^{-}\right]$and $\left[1011 / 2^{-}\right]$that generate low-lying peaks cannot be descried properly by the simple shift of the Gaussian basis.

The full GCM includes all of the basis wave functions which are the single-particle excited states obtained by the energy variation and the various $1 p 1 h$ configurations generated by the shifted Gaussian basis. Therefore, I expect both of the collective and single-particle excitations are reasonably described. The strength function obtained by the full GCM is shown by the orange histogram and red line in Fig. 4(b). It has two peaked GDR distribution similar to the shifted-GCM and low-lying strengths around 5-10 $\mathrm{MeV}$ which should be attributed to the pygmy dipole resonance. The calculated energy weighted sum and GDR energy are similar to the result of the shifted-basis GCM and other theoretical calculations.

Finally, I examine the convergence of the full GCM calculation. If the model space spanned by the shifted-basis

TABLE IV. Energy weighted sum in $e^{2} \mathrm{fm}^{2} \mathrm{MeV}$ and centroid energy of GDR (peak position and the ratio of energy weighted and nonweighted sums) in MeV obtained by $\beta$ GCM, shifted-basis GCM, and full GCM. They are compared with the QRPA calculations [16,21] which also use Gogny D1S interaction.

\begin{tabular}{lrcc}
\hline \hline & $m_{1}$ & $m_{1} / m_{0}$ & Peak position \\
\hline$\beta$ GCM & 5 & 11 & \\
Shifted-basis GCM $(\epsilon=0.30)$ & 177 & 24.5 & 22.0 \\
Full GCM $(\epsilon=0.30)$ & 183 & 23.4 & 21.5 \\
Full GCM $(\epsilon=0.20)$ & 176 & 22.7 & 21.5 \\
Full GCM $(\epsilon=0.40)$ & 171 & 23.1 & 21.5 \\
Peru et al. $[16]$ & & & 21.9 \\
Hashimoto [21] & 181 & 24.5 & 22.5 \\
\hline \hline
\end{tabular}

functions is large enough and if the magnitude of the shift $\epsilon$ is small enough, the result should not depend on the magnitude of $\epsilon$. To investigate the convergency, I performed full GCM calculations by changing the magnitude of the $\epsilon$ to 0.2 and $0.4 \mathrm{fm}$ as shown in Fig. 4(b). It is clear that the strength distribution below $25 \mathrm{MeV}$ is almost unchanged, while the peak around $28 \mathrm{MeV}$ is slightly affected. Therefore, I conclude that the result for the pygmy dipole resonance and the first lower peak of GDR is well converged, while the higher peak of GDR is somewhat ambiguous. I also note that the energy weight sum of the strength and the centroid energy of GDR are rarely affected by the choice of $\epsilon$ as shown in Table IV.

\section{DISCUSSIONS}

Here, I first focus on the high-energy part of the calculated $E 1$ response and discuss the splitting of the GDR and its relationship to the ground-state deformation. Then, I discuss the low-energy part, i.e., the PDR and analyze its characteristics.

\section{A. Splitting of GDR}

It is well known that the ground-state deformation affects the distribution of the giant resonances. In the case of the $E 1$ response of axially symmetric nucleus, the ground-state deformation differentiates the oscillator length for the collective vibration along the longest and shortest deformation axes, which results in the splitting of the GDR into two components. The QRPA calculation $[18,56]$ shown that the $K^{\pi}=0^{-}$ component of the GDR appears at smaller excitation energy than the $K^{\pi}= \pm 1^{-}$component for the prolate deformed nuclei, while the order is inverted in the oblate deformed nuclei [57].

However, the discussion made by QRPA calculations is based on the analysis in the body-fixed frame where the deformed intrinsic state is not an eigenstate of good angular momentum, and hence, the calculated results do not directly correspond to the observed excitation function of $1^{-}$states. On the other hand, in the present calculation, the results can be directly compared with the observed data, because the 


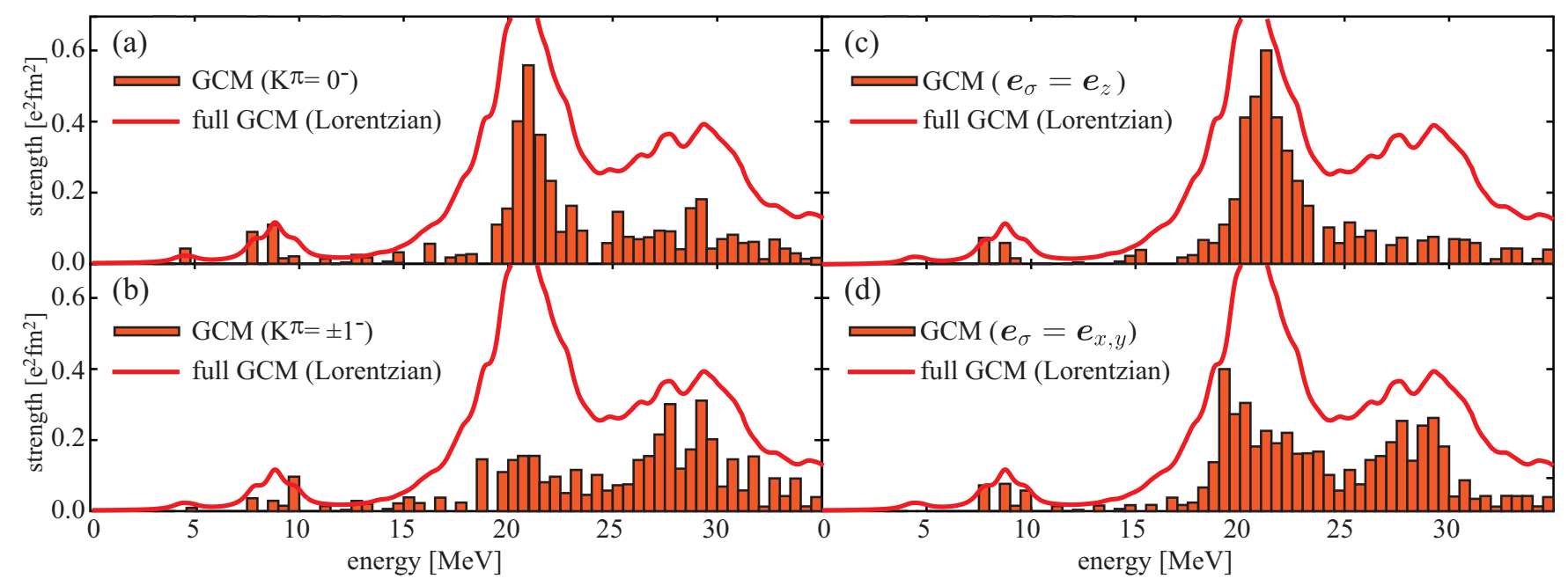

FIG. 5. The electric dipole strength functions of ${ }^{26} \mathrm{Ne}$ obtained by the GCM calculations with the limited model spaces. (a) and (b) The $K$ quantum numbers are limited to $K=0$ or $K= \pm 1$. (c) and (d) The directions of the shift is limited to only $z$ or $x, y$ directions.

rotational symmetry is restored by the angular momentum projection. Because the excitation function shown in Fig. 4 also shows the splitting of GDR, it is of interest to check if it really originates in the ground-state deformation or not. For this purpose, I have performed two additional GCM calculations. In the first calculation, the value of the $K$ quantum number is restricted to $K=0$ (or \pm 1$)$ in the GCM calculation. In other words, the summation over $K$ in Eq. (21) is restricted to only $K=0$ or $K= \pm 1$, which will distinguish the $K=0$ and \pm 1 components. In the second calculation, the value of the $K$ quantum number is unrestricted, but the shift of Gaussian centroids [the unit vector $\boldsymbol{e}$ in Eq. (18)] is restricted to only the $z$ direction (or $x$ and $y$ directions) where the $z$ axis is chosen to be the longest deformation axis. This will apparently restrict the direction of the vibration to the $z$ ( $x$ and $y$ ) direction. The results of the calculations are presented in Fig. 5. As clearly seen, both calculations show that the low-energy part of GDR is dominated by the vibration along the longest deformation axis $\left(K^{\pi}=0^{-}\right.$and $\left.\boldsymbol{e}=\boldsymbol{e}_{z}\right)$, while the high-energy part is dominated by the vibration along the shortest deformation axis $\left(K^{\pi}= \pm 1^{-}\right.$and $\left.\boldsymbol{e}=\boldsymbol{e}_{x, y}\right)$.

Thus, the splitting of the vibration modes parallel and perpendicular to the longest axis in the intrinsic frame also can be observed even after the angular momentum projection. Hence I can safely conclude that the splitting of GDR surely originates in the ground-state deformation. It is also noted that the low (high) energy part of PDR is also dominated by the $K^{\pi}=0^{-}\left( \pm 1^{-}\right)$component, which is also qualitatively consistent with the QRPA result [18].

\section{B. Property of PDR}

Figure 6 magnifies the low-energy part of the strength function where six $1^{-}$states locate in between 4 and $10 \mathrm{MeV}$ as listed in Table V. Among them, I regard that the four $1^{-}$ states $\left(1_{3}^{-}\right.$to $\left.1_{6}^{-}\right)$which locate above the neutron threshold (5.5 MeV) correspond to the observed PDR, because the experiment [14] was sensitive to only the neutron decaying states. The averaged energy of these four $1^{-}$states is $8.5 \mathrm{MeV}$ and the sum of $B(E 1)$ is $0.44 e^{2} \mathrm{fm}^{2}$, which reasonably agrees with the observed data, $9 \mathrm{MeV}$ and $0.49 \pm 0.16 e^{2} \mathrm{fm}^{2}$. The energy weighted sum amounts to approximately $4 \%$ of the TRK sum rule, which is slightly smaller than the observation which amounts to approximately 5\%.

Table VI suggests that there are several interesting features to be noted in the calculated $\mathrm{S}$ factors of the low-lying $1^{-}$states. First, the $\mathrm{S}$ factors of the $1_{1}^{-}$and $1_{2}^{-}$states are large in the ground-state channels $\left[{ }^{25} \mathrm{Ne}\left(1 / 2_{1}^{+}\right) \otimes l_{j}\right]$. On the other hand, those of the $1_{3}^{-}$to $1_{6}^{-}$states are small in the ground-state channels but large in the excited state channels $\left[{ }^{25} \mathrm{Ne}\left(5 / 2_{1}^{+}\right) \otimes l_{j},{ }^{25} \mathrm{Ne}\left(3 / 2_{1}^{+}\right) \otimes l_{j}\right.$, and $\left.{ }^{25} \mathrm{Ne}\left(3 / 2_{1}^{-}\right) \otimes l_{j}\right]$ indicating that the PDR of ${ }^{26} \mathrm{Ne}$ involves the core excitation. This may be a straightforward answer to the question, "Why does the observed ${ }^{26} \mathrm{Ne}$ PDR predominantly decay to the excited state of ${ }^{25} \mathrm{Ne}$, not to its ground state?" The reason for the core excitation may be attributed to the deformation of PDR. In the strong coupling picture, it can be easily shown that PDR has a large amount of the core excited component. Another possible reason is the isoscalar component in PDR. I'll discuss, in the next section, that the large IS component in PDR possibly induces strong quadrupole core excitation.

The second is the dominance of the $p_{3 / 2} \mathrm{~S}$ factors over the $f_{7 / 2} \mathrm{~S}$ factors. There may be several explanations for this.

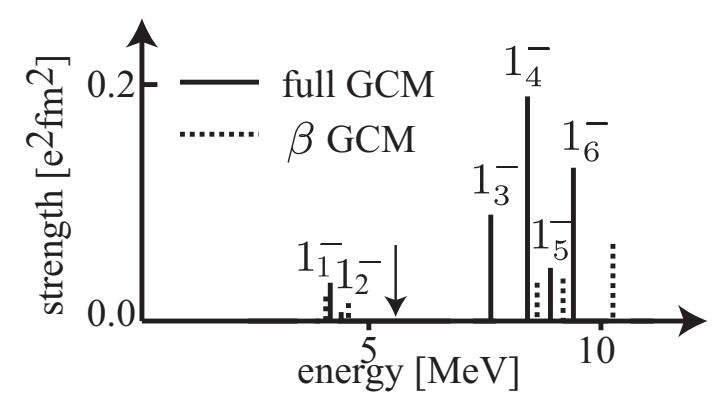

FIG. 6. Distributions of the low-lying $E 1$ strengths calculated by full GCM and $\beta$ GCM. Arrow shows the neutron threshold. 
TABLE V. Energies and $B(E 1)$ strengths of four $1^{-}$states $\left(1_{3}^{-}\right.$to $\left.1_{6}^{-}\right)$which constitute the PDR. Their averaged energy weighted by $B(E 1)$ strength and the sum of the $B(E 1)$ strength are compared with the observed data [14].

\begin{tabular}{lcc}
\hline \hline & $E_{x}(\mathrm{MeV})$ & $B\left(E 1 ; 0_{1}^{+} \rightarrow 1^{-}\right)\left(e^{2} \mathrm{fm}^{2}\right)$ \\
\hline $1_{1}^{-}$ & 4.0 & 0.03 \\
$1_{2}^{-}$ & 4.6 & 0.01 \\
$1_{3}^{-}$ & 7.6 & 0.09 \\
$1_{4}^{-}$ & 8.4 & 0.19 \\
$1_{5}^{-}$ & 8.9 & 0.04 \\
$1_{6}^{-}$ & 9.4 & 0.12 \\
Total $\left(1_{3}^{-}\right.$to $\left.1_{6}^{-}\right)$ & 8.5 & 0.44 \\
Expt. & 9 & $0.49 \pm 0.16$ \\
\hline \hline
\end{tabular}

The first reason is derived from a simple spherical shell model picture. In the spherical shell model, the last neutron occupies $1 s_{1 / 2}$ in the ground state. This last neutron must be excited to $p_{3 / 2}$ not to $f_{7 / 2}$ to generate the $1^{-}$state. The second is given by the deformation picture. As already shown, the PDR has a large amount of the $v\left[3301 / 2^{-}\right]$component. As is well known, as deformation becomes larger, this Nilsson orbit has a large contamination of $p_{3 / 2}$. The final explanation is the quenching of the $N=28$ shell gap. It was discussed that the quenching of the $N=28$ shell gap also strongly affects the neutron-rich $\mathrm{Ne}$ and $\mathrm{Mg}$ isotopes in the island of inversion where the $N=20$ shell gap is broken. A well-known famous example is the neutron-halo nucleus ${ }^{31} \mathrm{Ne}$ with $N=21$, in which the ground state has the $v p_{3 / 2}$ configuration instead of $v f_{7 / 2}$. Even in the case of ${ }^{26} \mathrm{Ne}$ which is out of the island of inversion, the quenching of the $N=28$ shell gap will affect the excitation spectra. Indeed, one is reminded that the $3 / 2^{-}$state is lower than the $7 / 2^{-}$state in ${ }^{25} \mathrm{Ne}$.

\section{Isoscalar component of PDR}

The dominance of the core excitation discussed above implies that the PDR has large isoscalar (IS) dipole strength

TABLE VI. Single-particle spectroscopic factors in ${ }^{25} \mathrm{Ne}\left(J^{\pi}\right) \otimes$ $\ell_{j}$ channels for the low-lying $1^{-}$states.

\begin{tabular}{ccccc}
\hline \hline & $1 / 2_{1}^{+} \otimes p_{3 / 2}$ & $1 / 2_{1}^{+} \otimes p_{1 / 2}$ & $3 / 2_{1}^{+} \otimes p_{3 / 2}$ & $3 / 2_{1}^{+} \otimes p_{1 / 2}$ \\
\hline $1_{1}^{-}$ & 0.8 & 0.3 & 0.2 & 0.0 \\
$1_{2}^{-}$ & 0.2 & 0.7 & 0.0 & 0.3 \\
$1_{3}^{-}$ & 0.1 & 0.1 & 0.4 & 0.0 \\
$1_{4}^{-}$ & 0.3 & 0.0 & 0.3 & 0.1 \\
$1_{5}^{-}$ & 0.2 & 0.0 & 0.2 & 0.1 \\
$1_{6}^{-}$ & 0.1 & 0.1 & 1.1 & 0.2 \\
& $5 / 2_{1}^{+} \otimes p_{3 / 2}$ & $5 / 2_{1}^{+} \otimes f_{7 / 2}$ & $3 / 2_{1}^{-} \otimes s_{1 / 2}$ & $3 / 2_{1}^{-} \otimes d_{3 / 2}$ \\
\hline $1_{1}^{-}$ & 0.0 & 0.1 & 0.2 & 0.0 \\
$1_{2}^{-}$ & 0.0 & 0.3 & 0.0 & 0.1 \\
$1_{3}^{-}$ & 1.2 & 0.9 & 0.4 & 0.2 \\
$1_{4}^{-}$ & 1.1 & 0.6 & 0.3 & 0.1 \\
$1_{5}^{-}$ & 0.3 & 0.1 & 0.7 & 0.4 \\
$1_{6}^{-}$ & 0.2 & 0.1 & 0.5 & 0.3 \\
\hline \hline
\end{tabular}

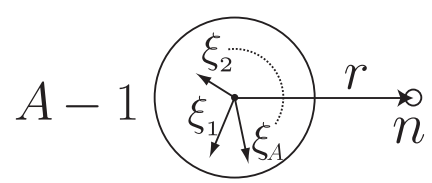

FIG. 7. Schematic figure for the internal coordinates $\boldsymbol{\xi}_{i}$ and the relative coordinate $\boldsymbol{r}$.

as well as the IV strength. This is explained as follows. The first line of Eq. (32) is the standard definition of the IS dipole transition operator in terms of the single-particle coordinate $\boldsymbol{r}_{i}$ and the center-of-mass coordinate $\boldsymbol{r}_{\mathrm{c} . \mathrm{m} .}=\sum_{i=1}^{A} \boldsymbol{r}_{i} / A$.

$$
\begin{aligned}
\mathcal{M}_{\mu}(\mathrm{IS} 1)= & \sum_{i=1}^{A}\left(\boldsymbol{r}_{i}-\boldsymbol{r}_{\mathrm{c} . \mathrm{m} .}\right)^{2} \mathcal{Y}_{1 \mu}\left(\boldsymbol{r}_{i}-\boldsymbol{r}_{\mathrm{c} . \mathrm{m} .}\right) \\
= & \sum_{i=1}^{A-1} \xi_{i}^{2} \mathcal{Y}_{1 \mu}\left(\boldsymbol{\xi}_{i}\right)+\frac{(A-1)(A-2)}{A^{2}} r^{2} \mathcal{Y}_{1 \mu}(\boldsymbol{r}) \\
& -\frac{5}{3 A} \sum_{i=1}^{A-1} \xi_{i}^{2} \mathcal{Y}_{1 \mu}(\boldsymbol{r}) \\
& +\frac{4 \sqrt{2 \pi}}{3 A}\left[\sum_{i=1}^{A-1} \mathcal{Y}_{2}\left(\boldsymbol{\xi}_{i}\right) \otimes \mathcal{Y}_{1}(\boldsymbol{r})\right]_{1 \mu} \\
\simeq & \frac{4 \sqrt{2 \pi}}{3 A}\left[\sum_{i=1}^{A-1} \mathcal{Y}_{2}\left(\boldsymbol{\xi}_{i}\right) \otimes \mathcal{Y}_{1}(\boldsymbol{r})\right]_{1 \mu}
\end{aligned}
$$

Then, I divide the system into the core nucleus with mass $A-1$ and the valence neutron, and introduce the internal coordinate of the core $\boldsymbol{\xi}_{i}$ and the relative coordinate between the core and the valence neutron $\boldsymbol{r}$ (see Fig. 7),

$$
\begin{aligned}
\boldsymbol{\xi}_{i} & =\boldsymbol{r}_{i}-\frac{1}{A-1} \sum_{i=1}^{A-1} \boldsymbol{r}_{i}, \quad i=1,2, \ldots, A-1, \\
\boldsymbol{r} & =r_{A}-\frac{1}{A-1} \sum_{i=1}^{A-1} \boldsymbol{r}_{i} .
\end{aligned}
$$

Using these coordinates, the operator is equivalently rewritten as the second line of Eq. (32) (see Appendix C and Ref. [58]). Now I examine each term of the second line. The first term is the IS dipole excitation of the core nucleus and should have only negligible contribution to PDR, because it involves the change of the core density, and hence, it cannot contribute to the low-energy excitation modes. The second term is the dipole excitation of the relative motion between the core and the valence neutron. The third term is also the dipole excitation of the relative motion, but it is coupled to the monopole operator of the core. One can also expect that these two terms cancel out to each other and their contribution can be negligible. To elucidate it, let us simplify the wave functions of the ground state and PDR as

$$
\begin{aligned}
|\mathrm{GS}\rangle & =\left|\Phi_{C}\right\rangle\left|\phi_{n}\right\rangle, \\
|\mathrm{PDR}\rangle & =\sqrt{1-c^{2}}\left|\Phi_{C}\right\rangle\left|\phi_{n}^{*}\right\rangle+c\left|\Phi_{C}^{*}\right\rangle\left|\phi_{n}^{* *}\right\rangle,
\end{aligned}
$$


where $\left|\Phi_{C}\right\rangle$ and $\left|\Phi_{C}^{*}\right\rangle$ are the ground and excited state wave functions of the core. $\left|\phi_{n}\right\rangle$ is the valence neutron in the ground state, while $\left|\phi_{n}^{*}\right\rangle$ and $\left|\phi_{n}^{* *}\right\rangle$ are those in the PDR coupled to the ground and excited states of the core. The antisymmetrization between the core and the valence neutron is neglected for simplicity. Using these wave functions, the IS dipole transition matrix between the ground state and PDR may be estimated as follows. The second term of Eq. (32) yields

$$
\frac{(A-1)(A-2)}{A^{2}} \sqrt{1-c^{2}}\left\langle\phi_{n}^{*}\left|r^{2} \mathcal{Y}_{1 \mu}(\boldsymbol{r})\right| \phi_{n}\right\rangle,
$$

and the third term contribution is

$$
-\frac{5}{3} \frac{A-1}{A} \sqrt{1-c^{2}}\left\langle r_{C}^{2}\right\rangle\left\langle\phi_{n}^{*}\left|\mathcal{Y}_{1 \mu}(\boldsymbol{r})\right| \phi_{n}\right\rangle .
$$

Here, I assumed that $\left|\Phi_{C}\right\rangle$ and $\left|\Phi_{C}^{*}\right\rangle$ have different angular momenta, and hence, $\left\langle\Phi_{C}^{*} \mid \Phi_{C}\right\rangle=\left\langle\Phi_{C}^{*}\left|\sum_{i=1}^{A-1} \xi_{i}^{2}\right| \Phi_{C}\right\rangle=0$. $\left\langle r_{C}^{2}\right\rangle$ denotes the mean-square radius of the core ground state, i.e., $\left\langle r_{C}^{2}\right\rangle=\left\langle\Phi_{C}\left|\sum_{i=1}^{A-1} \xi_{i}^{2}\right| \Phi_{C}\right\rangle /(A-1)$. If the radius of the core $\left|\Phi_{C}\right\rangle$ and that of the valence neutron $\left|\phi_{n}\right\rangle$ are almost the same size, one might expect that the matrix elements $\left\langle\phi_{n}^{*}\left|r^{2} \mathcal{Y}_{1 \mu}(\boldsymbol{r})\right| \phi_{n}\right\rangle$ and $\left\langle r_{C}^{2}\right\rangle\left\langle\phi_{n}^{*}\left|\mathcal{Y}_{1 \mu}(\boldsymbol{r})\right| \phi_{n}\right\rangle$ are the same order of magnitude. Hence, one would expect that the second and third terms largely cancel out each other for such a situation [59].

Thus, I expect that only the fourth term has the sizable contribution to the low-lying dipole mode as written in the last line of Eq. (32). It is the dipole excitation of the valence neutron coupled to the quadrupole operator of the core. Assuming that the ground state of the core has no quadrupole moment (this is true for the $1 / 2^{+}$ground state of ${ }^{25} \mathrm{Ne}$ ), the contribution from the fourth term is estimated as

$$
\frac{4 \sqrt{2 \pi}}{3 A} c\left\langle Q_{C}\right\rangle\left\langle\phi_{n}^{* *}\left|r \mathcal{Y}_{1 \mu}(\boldsymbol{r})\right| \phi_{n}\right\rangle,
$$

where the quadrupole matrix element of the core is defined as $\left\langle Q_{C}\right\rangle=\left\langle\Phi_{C}^{*}\left|\sum_{i=1}^{A} \mathcal{Y}_{2 \mu}\left(\xi_{i}\right)\right| \Phi_{C}\right\rangle$. Remember that the first and second excited states of ${ }^{25} \mathrm{Ne}$ have large $B(E 2)$ value (Table II). Hence the matrix element $\left\langle Q_{C}\right\rangle$ should be large and the fourth term should yield a large IS dipole transition matrix. In other words, the IS dipole transition is sensitive to the quadrupole excitation of the core, and the PDR of ${ }^{26} \mathrm{Ne}$ should have a large IS dipole transition matrix if the core excited component is important as discussed in Sec. IV B.

The above discussion is based on many assumptions, and must be verified by the numerical calculation without approximations. Figure 8(a) shows the calculated IS dipole strength. Note that the results shown in Fig. 8(a) are obtained from the full GCM wave function without any truncation of the IS dipole operator and wave functions. One clearly sees that the PDR has pronounced IS dipole strength as expected from the above discussion. To make the argument more visible, Fig. 8(c) shows the IS dipole strength of the excited state that has the sizable $E 1$ strengths $\left(B(E 1)>0.05 e^{2} \mathrm{fm}^{2}\right)$. It is obvious that only the PDR has large $E 1$ and IS dipole strengths simultaneously, while the other excited states do not. Thus, the IS dipole strength is correlated well with the core excitation of PDR and its mechanism may be explained by Eq. (32). I

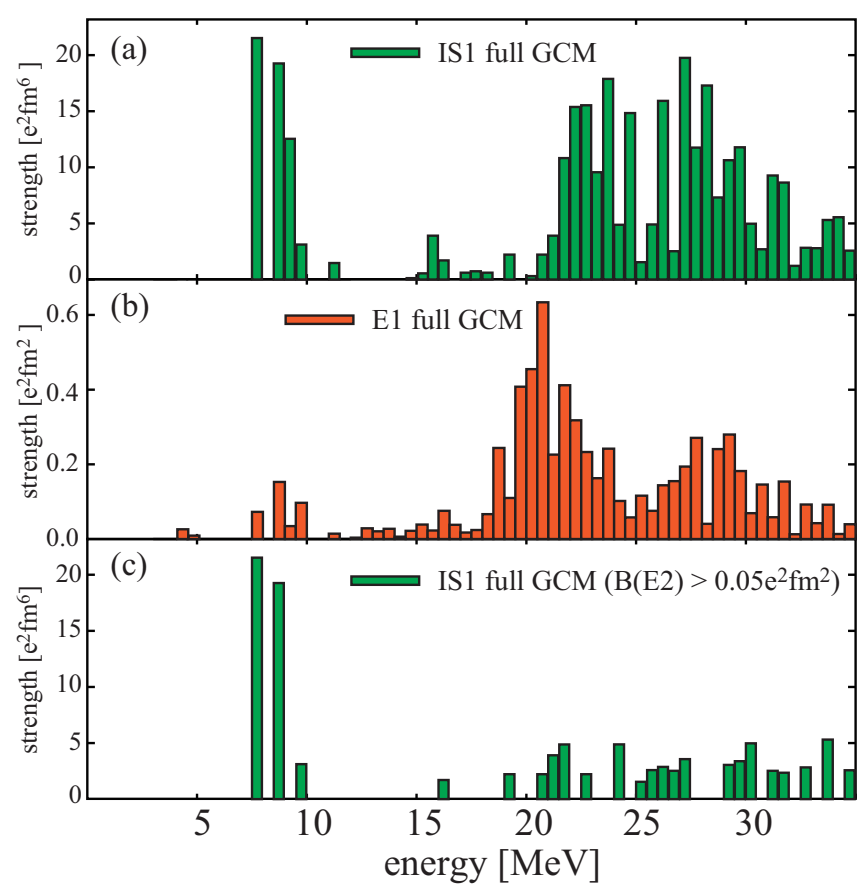

FIG. 8. (a) Calculated IS dipole strength distribution. (b) Calculated electric dipole strength distribution which is the same as Fig. 4(a). (c) Calculated IS dipole strengths of the states having sizable magnitude of $E 1$ strengths $\left[B(E 1)>0.05 e^{2} \mathrm{fm}^{2}\right]$.

expect that IS dipole strength will provide good insight into the structure of PDR, if it is experimentally measured.

Knowing the above-mentioned results, one may also be able to conjecture as follows. Imagine that the PDR is dominated by the neutron single-particle excitation and proton excitation plays only a minor role. In such cases, the PDR is not an eigenmode of the isospin, but a mixture of the IV and IS components,

$$
|\mathrm{PDR}\rangle \propto \mathcal{M}(E 1)|\mathrm{GS}\rangle+\mathcal{M}(I S 1)|G S\rangle .
$$

Indeed, this kind of contamination of the isoscalar component has already been discussed by many authors [60-63]. Then, suppose that the core nucleus has strong low-lying quadrupole collectivity. From the above discussions, one can expect that $\mathcal{M}(I S 1)|G S\rangle$ is strongly amplified, and as a result, the PDR is predominated by the core excited component. In short, I conjecture that the PDR will be dominated by the core excited component, if the core nucleus has low-lying strong quadrupole collectivity. A good candidate of this conjecture is neutron-rich $\mathrm{Ne}$ isotopes which have very strong quadrupole collectivity owing to the breakdown of the $N=20$ magic number in the island of inversion. This conjecture will be tested by the undergoing numerical calculations.

\section{SUMMARY}

In summary, I have investigated the pygmy dipole resonance of ${ }^{26} \mathrm{Ne}$ by using the shifted-basis AMD. The ordinary AMD framework, $\beta$ GCM, reasonably described the low-lying spectra of ${ }^{24,25,26} \mathrm{Ne}$, but failed to describe the $E 1$ response of ${ }^{26} \mathrm{Ne}$. The shifted-basis AMD introduces various $1 \mathrm{p} 1 \mathrm{~h}$ 
configurations by the shift of the nucleon wave packets and is able to describe the $E 1$ response. The global feature of the calculated $E 1$ response function was consistent with the QRPA calculations which employ the same Gogny D1S interaction. It also showed that the splitting of the GDR originates in the ground-state deformation.

The shifted-basis AMD showed that the PDR appears approximately at $8.5 \mathrm{MeV}$ and exhausts $4 \%$ of the TRK sum which is consistent with the observation. The structure of the PDR was examined by the analysis of the spectroscopic factors. It was found that the PDR is dominated by the neutron excitation coupled to the quadrupole excited core nucleus ${ }^{25} \mathrm{Ne}$, which explains the observed decay of PDR to the excited states of ${ }^{25} \mathrm{Ne}$. I suggested that the quadrupole core excitation induces the large contamination of the isoscalar component in PDR. It was shown by the analytic calculation by rewriting the isoscalar dipole operator in terms of the internal coordinates and the relative coordinate between the core and the valence neutron. This estimation was confirmed by the numerical calculation using shifted-basis AMD. From this result, I conjecture that the PDR will be dominated by the core excited component, if the core nucleus has low-lying strong quadrupole collectivity. By the undergoing numerical calculations, this conjecture will be tested in neutron-rich $\mathrm{Ne}$ isotopes in which the low-lying strong quadrupole collectivity is well known.

\section{ACKNOWLEDGMENTS}

The author acknowledges the fruitful discussions with Dr. Kanada-En'yo. He also acknowledges support from the Grants-in-Aid for Scientific Research on Innovative Areas from MEXT (Grant No. 2404:24105008) and JSPS KAKENHI Grant No. 16K05339. Part of the numerical calculations were performed by using the super computer in Yukawa Institute for Theoretical Physics (YITP) in Kyoto University.

\section{APPENDIX A: OVERLAP AMPLITUDE AND SINGLE-PARTICLE SPECTROSCOPIC FACTOR OF SLATER DETERMINANTS}

In this Appendix, I derive the equations to calculate the overlap amplitude and spectroscopic factor. The following equations are applicable for nuclear models based on Slater determinant wave functions such as Hartree-Fock as well as AMD.

I first consider the Slater determinant wave functions of $A$ and $A+1$ body systems given as

$$
\begin{aligned}
\Phi\left(\boldsymbol{r}_{1}, \ldots, \boldsymbol{r}_{A}\right) & =\frac{1}{\sqrt{A !}} \operatorname{det}\left\{\phi_{1} \cdots \phi_{A}\right\}, \\
\Psi\left(\boldsymbol{r}_{1}, \ldots, \boldsymbol{r}_{A+1}\right) & =\frac{1}{\sqrt{(A+1) !}} \operatorname{det}\left\{\psi_{1} \cdots \psi_{A+1}\right\} .
\end{aligned}
$$

Using the $A \times(A+1)$ overlap matrix $B_{i j}=\left\langle\phi_{i} \mid \psi_{j}\right\rangle$ and its submatrix $B^{(p)}$ formed by removing the $p$ th column from $B$, the overlap amplitude is calculated as

$$
\begin{aligned}
& \varphi(\boldsymbol{r}) \equiv \sqrt{A+1}\langle\Phi \mid \Psi\rangle
\end{aligned}
$$

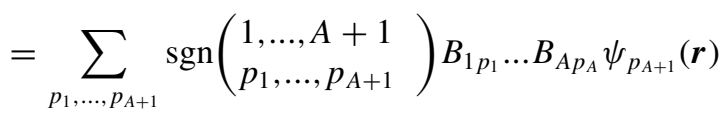

$$
\begin{aligned}
& =\sum_{p=1}^{A+1}(-)^{p} \operatorname{det} B^{(p)} \psi_{p}(\boldsymbol{r}),
\end{aligned}
$$

where a trivial factor $(-)^{A+1}$ is omitted for simplicity. Using this result, I consider the overlap amplitude of angular momentum projected Slater determinants $P_{M K}^{J} \Phi$ and $P_{M K}^{J} \Psi$. Their overlap amplitude is calculated as follows.

$$
\begin{aligned}
\varphi(\boldsymbol{r}) & \equiv \sqrt{A+1}\left\langle P_{M_{2} K_{2}}^{J_{2}} \Phi \mid P_{M_{1} K_{1}}^{J_{1}} \Psi\right\rangle=\frac{\left(2 J_{1}+1\right)\left(2 J_{2}+1\right)}{\left(8 \pi^{2}\right)^{2}} \int d \Omega_{1} d \Omega_{2} D_{M_{2} K_{2}}^{J_{2}}\left(\Omega_{2}\right) D_{M_{1} K_{1}}^{J_{1} *}\left(\Omega_{1}\right) \sqrt{A+1}\left\langle R_{A}\left(\Omega_{2}\right) \Phi \mid R_{A+1}\left(\Omega_{1}\right) \Psi\right\rangle \\
& =\frac{\left(2 J_{1}+1\right)\left(2 J_{2}+1\right)}{\left(8 \pi^{2}\right)^{2}} \sum_{K} \int d \Omega_{1} d \Omega_{2}^{\prime} D_{M_{2} K}^{J_{2}}\left(\Omega_{1}\right) D_{K K_{2}}^{J_{2}}\left(\Omega_{2}^{\prime}\right) D_{M_{1} K_{1}}^{J_{1} *}\left(\Omega_{1}\right) \sqrt{A+1}\left\langle\Phi\left|R_{A}^{\dagger}\left(\Omega_{2}^{\prime}\right) R_{A}^{\dagger}\left(\Omega_{1}\right) R_{A+1}\left(\Omega_{1}\right)\right| \Psi\right\rangle, \quad(\mathrm{A} 4)
\end{aligned}
$$

where $\Omega_{2}^{\prime}$ satisfies the relation $R\left(\Omega_{2}\right)=R\left(\Omega_{1}\right) R\left(\Omega_{2}^{\prime}\right)$, and hence, $D_{M_{2} K_{2}}^{J_{2}}\left(\Omega_{2}\right)=\sum_{K} D_{M_{2} K}^{J_{2}}\left(\Omega_{1}\right) D_{K K_{2}}^{J_{2}}\left(\Omega_{2}^{\prime}\right)$. Note that $R_{A}(\Omega)$ rotates $\boldsymbol{r}_{1}, \ldots, \boldsymbol{r}_{A}$, while $R_{A+1}(\Omega)$ rotates $\boldsymbol{r}_{1}, \ldots, \boldsymbol{r}_{A+1}$. Then, using Eq. (C2), the bracket in the integral is calculated.

$$
\sqrt{A+1}\left\langle\Phi\left|R_{A}^{\dagger}\left(\Omega_{2}^{\prime}\right) R_{A}^{\dagger}\left(\Omega_{1}\right) R_{A+1}\left(\Omega_{1}\right)\right| \Psi\right\rangle=\sum_{p=1}^{A+1}(-)^{p} \operatorname{det} B^{(p)}\left(-\Omega_{2}^{\prime}\right)\left\{R\left(\Omega_{1}\right) \psi_{p}\left(\boldsymbol{r}_{A+1}\right)\right\} .
$$

Here, $B^{(p)}\left(-\Omega_{2}^{\prime}\right)$ is a $A \times A$ submatrix of $B_{i j}\left(-\Omega_{2}^{\prime}\right)=\left\langle\phi_{i}\left|R^{\dagger}\left(\Omega_{2}\right)\right| \psi_{j}\right\rangle$.

Now using the multipole expansion,

$$
\psi_{p}(\boldsymbol{r})=\sum_{j l k} \psi_{j l k}^{(p)}(r)\left[Y_{l}(\hat{r}) \otimes \chi\right]_{j k}
$$

the rotation of $\psi_{p}(\boldsymbol{r})$ is written as

$$
R\left(\Omega_{1}\right) \psi_{p}(\boldsymbol{r})=\sum_{j l m k} \psi_{j l m}^{(p)}(r) D_{m k}^{j}\left(\Omega_{1}\right)\left[Y_{l}(\hat{r}) \otimes \chi\right]_{j m}
$$


Substituting Eqs. (A5) and (A7) into Eq. (C6), the integral over $\Omega_{1}$ is analytically performed. Simplifying the equation, one obtains the overlap amplitude for the angular momentum projected Slater determinants.

$$
\begin{gathered}
\varphi(\boldsymbol{r})=\sqrt{A+1}\left\langle P_{M_{2} K_{2}}^{J_{2}} \Phi \mid P_{M_{1} K_{1}}^{J_{1}} \Psi\right\rangle=\sum_{j l} C_{J_{2} M_{2}, j M_{1}-M_{2}}^{J_{1} M_{1}} \varphi_{j l}(r)\left[Y_{l}(\hat{r}) \otimes \chi\right]_{j M_{1}-M_{2}}, \\
\varphi_{j l}(r)=\sum_{k} C_{J_{2} K_{1}-k, j k}^{J_{1} K_{1}} \sum_{p=1}^{A+1}(-)^{p} \psi_{j l k}^{(p)}(r) \frac{2 J_{2}+1}{8 \pi^{2}} \int d \Omega D_{K_{2} K_{1}-k}^{J_{2} *}(\Omega) \operatorname{det} B^{(p)}(\Omega),
\end{gathered}
$$

where $C_{j_{1} m_{1}, j_{2} m_{2}}^{J M}$ denotes Clebsch-Gordan coefficient. It is obvious that the overlap amplitude of the GCM wave functions given in Eqs. (12), (20), and (21) are obtained by a linear transformation of Eq. (A9).

By a similar manner calculation, the equation for two-body overlap amplitude $\varphi\left(\boldsymbol{r}_{1}, \boldsymbol{r}_{2}\right)$ for $A$ and $A+2$ body systems is also obtained as follows.

$$
\begin{aligned}
\varphi\left(\boldsymbol{r}_{1}, \boldsymbol{r}_{2}\right) & \equiv \sqrt{(A+1)(A+2)}\left\langle P_{M_{2} K_{2}}^{J_{2}} \Phi \mid P_{M_{1} K_{1}}^{J_{1}} \Psi\right\rangle \\
& =\sum_{j} C_{J_{2} M_{2}, j M_{1}-M_{2}}^{J_{1} M_{1}} \sum_{j_{1} l_{1} j_{2} l_{2}} \varphi_{j ; j_{1} l_{1} j_{2} l_{2}}\left(r_{1}, r_{2}\right)\left[\left[Y_{l_{1}}\left(\hat{r}_{1}\right) \otimes \chi_{1}\right]_{j_{1}} \otimes\left[Y_{l_{2}}\left(\hat{r}_{2}\right) \otimes \chi_{2}\right]_{j_{2}}\right]_{j M_{1}-M_{2},}, \\
\varphi_{j ; j_{1} l_{1} j_{2} l_{2}}\left(r_{1}, r_{2}\right) & =\sum_{k} C_{J_{2} K_{1}-k, j k}^{J_{1} K_{1}} \sum_{p<q}^{A+2}(-)^{p-q} \varphi_{j k ; j_{1} l_{1} j_{2} l_{2}}^{(p, q)}\left(r_{1}, r_{2}\right) \frac{2 J_{2}+1}{8 \pi^{2}} \int d \Omega D_{K_{2}, K_{1}-k}^{J_{2} *}(\Omega) \operatorname{det} B^{(p, q)}(\Omega), \\
\varphi_{j k ; j_{1} l_{1} j_{2} l_{2}}^{(p, q)}\left(r_{1}, r_{2}\right) & =\sum_{k_{1}} C_{j_{1} k_{1}, j_{2} k-k_{1}}^{j k}\left\{\psi_{j_{1} l_{1} k_{1}}^{(p)}\left(r_{1}\right) \psi_{j_{2} l_{2} k-k_{1}}^{(q)}\left(r_{2}\right)-\psi_{j_{1} l_{1} k_{1}}^{(q)}\left(r_{1}\right) \psi_{j_{2} l_{2} k-k_{1}}^{(p)}\left(r_{2}\right)\right\},
\end{aligned}
$$

where $B^{(p, q)}(\Omega)$ is an $A \times A$ submatrix which is formed by removing $p$ and $q$ columns from the $A \times(A+2)$ matrix $B_{i j}(\Omega)=$ $\left\langle\phi_{i}|R(\Omega)| \psi_{j}\right\rangle$. A similar formula for the two-body overlap function was also derived in Ref. [64].

\section{APPENDIX B: SHIFT OF GAUSSIAN CENTROIDS AND ELECTRIC DIPOLE OPERATOR}

Here, I briefly explain why the shift of the Gaussian wave packets can efficiently describe various $1 p 1 h$ configurations which coherently contribute the electric dipole modes. The meaning of shifting the Gaussian centroid becomes clear when one rewrites the Gaussian wave packet given in Eq. (4) as the coherent state as

$$
\begin{aligned}
\varphi_{i}(\mathbf{r} ; \boldsymbol{Z}) & =\prod_{\sigma=x, y, z}\left(\frac{2 v_{\sigma}}{\pi}\right)^{\frac{1}{4}} e^{-v_{\sigma}\left(r_{\sigma}-\frac{Z_{i \sigma}}{\sqrt{v \sigma}}\right)^{2}+\frac{1}{2} Z_{\sigma}^{2}} \chi_{i} \xi_{i}, \\
& =\left\langle\boldsymbol{r}\left|e^{-Z^{2} / 2} e^{\boldsymbol{Z} \cdot \hat{\boldsymbol{a}}^{\dagger}}\right| 0\right\rangle \chi_{i} \xi_{i}=\langle\boldsymbol{r} \mid \boldsymbol{Z}\rangle \chi_{i} \xi_{i},
\end{aligned}
$$

where $\hat{\boldsymbol{a}}^{\dagger}=\left(\hat{a}_{x}^{\dagger}, \hat{a}_{y}^{\dagger}, \hat{a}_{z}^{\dagger}\right)$ is the creation operator of the harmonic oscillator with $\hbar \omega_{\sigma}=2 \hbar^{2} v_{\sigma} / m$ and $\boldsymbol{Z}=\left(Z_{x}, Z_{y}, Z_{z}\right)$. The shift of the centroid, $\boldsymbol{Z} \rightarrow \boldsymbol{Z}+\Delta \boldsymbol{Z}$, is written as

$$
\begin{aligned}
\varphi_{i}(\mathbf{r} ; \boldsymbol{Z}+\Delta \boldsymbol{Z}) & =\left\langle\boldsymbol{r}\left|e^{-(Z+\Delta Z)^{2} / 2} e^{(\boldsymbol{Z}+\Delta \boldsymbol{Z}) \cdot \hat{\boldsymbol{a}}^{\dagger}}\right| 0\right\rangle \chi_{i} \xi_{i} \\
& \propto\left\langle\boldsymbol{r}\left|e^{\Delta \boldsymbol{Z} \cdot \hat{\boldsymbol{a}}^{\dagger}}\right| \boldsymbol{Z}\right\rangle \chi_{i} \xi_{i}=e^{\Delta \boldsymbol{Z} \cdot \hat{\boldsymbol{a}}^{\dagger}} \phi_{i}(\boldsymbol{r} ; \boldsymbol{Z}) .
\end{aligned}
$$

Thus, by the shift of the centroid, the wave packets are coherently excited, and when $\Delta \boldsymbol{Z}$ is sufficiently small, it becomes a linear combination of 0 and $1 \hbar \omega$ excitations from the original wave packet. Therefore, when one of the wave packets of a Slater determinant is slightly shifted, it corresponds to the $1 \hbar \omega$ excitation from the original Slater determinant.

The shift is also closely related to the dipole response. Suppose that the dipole resonances are well approximated by the ground-state wave function multiplied by the $E 1$ operator, then it is rewritten as follows,

$$
\mid E 1 \text { resoance }\rangle \simeq \sum_{i=1}^{Z} \mathcal{Y}_{1 \mu}\left(\hat{r}_{i}\right)|\mathrm{GS}\rangle \simeq \frac{1}{\delta} \sum_{i=1}^{Z}\left(e^{\delta \mathcal{Y}_{1 \mu}\left(\hat{r}_{i}\right)}-1\right)|\mathrm{GS}\rangle \text {. }
$$

Here $\delta$ is assumed to be sufficiently small number. If $|G S\rangle$ is a Slater determinant of the Gaussian wave packets, $\sum_{i=1}^{Z} e^{\delta \mathcal{Y}_{1 \mu}\left(\hat{r}_{i}\right)}|\mathrm{GS}\rangle$ may be rewritten as

$$
\sum_{i=1}^{Z} e^{\delta \mathcal{Y}_{1 \mu}\left(\hat{r}_{i}\right)} \mathcal{A}\left\{\varphi_{1}, \ldots, \varphi_{A}\right\}=\sum_{i=1}^{Z} \mathcal{A}\left\{\varphi_{1}, \ldots, e^{\delta \mathcal{Y}_{1 \mu}(\hat{r})} \varphi_{i}, \ldots \varphi_{A}\right\},
$$

and here $e^{\delta \mathcal{Y}_{1 \mu}(\hat{r})} \varphi$ corresponds to the shift of the centroid. For example, in the case of $\mu=0$, it corresponds to the shift along the $z$ axis as follows:

$$
\begin{aligned}
e^{\delta \mathcal{Y}_{1 \mu}(\hat{r})} \varphi\left(\boldsymbol{r} ; \boldsymbol{Z}_{i}\right) & =e^{\sqrt{4 \pi / 3} \delta z} \varphi\left(\boldsymbol{r} ; \boldsymbol{Z}_{i}\right) \propto \varphi\left(\boldsymbol{r} ; \boldsymbol{Z}+\epsilon \boldsymbol{e}_{z}\right), \\
\epsilon & =\sqrt{\frac{4 \pi}{3}} \frac{\delta}{2 \sqrt{v}} .
\end{aligned}
$$

Thus, the dipole modes with small amplitude corresponds to the shift of the Gaussian wave packets and it generates various $1 p 1 h$ configurations.

\section{APPENDIX C: IS DIPOLE OPERATOR}

Here I derive the IS dipole operator given in Eq. (32). I assume that the single-particle coordinates $\boldsymbol{r}_{i}$ with $i=$ $1, \ldots, A-1$ are the coordinates of the core with mass $A-1$, 
while $\boldsymbol{r}_{A}$ is that of the valence neutron. Then, note that the following relation holds.

$$
\boldsymbol{r}_{i}-\boldsymbol{r}_{\mathrm{c} . \mathrm{m} .}= \begin{cases}\xi_{i}-\frac{1}{A} \boldsymbol{r}, & i=1, \ldots, A-1 \\ \frac{A-1}{A} \boldsymbol{r}, & i=A,\end{cases}
$$

where $\xi_{i}$ and $\boldsymbol{r}$ are the internal coordinates of the core and the relative coordinate between the core and valence neutron defined by Eqs. (33) and (34). Using this relation, the IS dipole operator is rewritten as follows:

$$
\begin{aligned}
\mathcal{M}_{\mu}^{I S 1} & =\sum_{i=1}^{A}\left(\boldsymbol{r}_{i}-\boldsymbol{r}_{\mathrm{c} . \mathrm{m} .}\right)^{2} \mathcal{Y}_{1 \mu}\left(\boldsymbol{r}_{i}-\boldsymbol{r}_{\mathrm{c} . \mathrm{m} .}\right)=\sum_{i=1}^{A-1}\left(\boldsymbol{\xi}_{i}-\frac{1}{A} \boldsymbol{r}\right)^{2} \mathcal{Y}_{1 \mu}\left(\boldsymbol{\xi}_{i}-\frac{1}{A} \boldsymbol{r}\right)+\left(\frac{A-1}{A} \boldsymbol{r}\right)^{2} \mathcal{Y}_{1 \mu}\left(\frac{A-1}{A} \boldsymbol{r}\right) \\
& =\sum_{i=1}^{A-1} \xi_{i}^{2} \mathcal{Y}_{1 \mu}\left(\boldsymbol{\xi}_{i}\right)-\frac{1}{A} \sum_{i=1}^{A-1} \xi_{i}^{2} \mathcal{Y}_{1 \mu}(\boldsymbol{r})-\frac{2}{A} \sum_{i=1}^{A-1}\left(\boldsymbol{\xi}_{i} \cdot \boldsymbol{r}\right) \mathcal{Y}_{1 \mu}\left(\boldsymbol{\xi}_{i}\right)+\frac{(A-1)(A-2)}{A^{2}} r^{2} \mathcal{Y}_{1 \mu}(\boldsymbol{r}),
\end{aligned}
$$

where the relations $\sum_{i=1}^{A-1} \boldsymbol{\xi}_{i}=\sum_{i=1}^{A-1} \mathcal{Y}_{1 \mu}\left(\boldsymbol{\xi}_{i}\right)=0$, and $\mathcal{Y}_{1 \mu}(\alpha \boldsymbol{a}+\beta \boldsymbol{b})=\alpha \mathcal{Y}_{1 \mu}(\boldsymbol{a})+\beta \mathcal{Y}_{1 \mu}(\boldsymbol{b})$ are used to derive the second line. Now using the identities,

$$
\begin{aligned}
& {\left[\mathcal{Y}_{1}(\boldsymbol{a}) \otimes \mathcal{Y}_{1}(\boldsymbol{b})\right]_{00}=-\frac{\sqrt{3}}{4 \pi} \boldsymbol{a} \cdot \boldsymbol{b},} \\
& {\left[\mathcal{Y}_{1}(\boldsymbol{a}) \otimes \mathcal{Y}_{1}(\boldsymbol{a})\right]_{1 \mu}=0,} \\
& {\left[\mathcal{Y}_{1}(\boldsymbol{a}) \otimes \mathcal{Y}_{1}(\boldsymbol{a})\right]_{2 \mu}=\sqrt{3 / 10 \pi} \mathcal{Y}_{2 \mu}(\boldsymbol{a}),}
\end{aligned}
$$

one finds that the third term in the last line of Eq. (C2) reads,

$$
\begin{aligned}
\left(\boldsymbol{\xi}_{i} \cdot \boldsymbol{r}\right) \mathcal{Y}_{1 \mu}\left(\boldsymbol{\xi}_{i}\right) & =-\frac{4 \pi}{\sqrt{3}}\left[\mathcal{Y}_{1}\left(\boldsymbol{\xi}_{i}\right) \otimes\left[\mathcal{Y}_{1}\left(\boldsymbol{\xi}_{i}\right) \otimes \mathcal{Y}_{1}(\boldsymbol{r})\right]_{0}\right]_{1 \mu}=-\frac{4 \pi}{\sqrt{3}} \sum_{l=0,1,2} \sqrt{2 l+1}\left\{\begin{array}{lll}
1 & 1 & l \\
1 & 1 & 0
\end{array}\right\}\left[\left[\mathcal{Y}_{1}\left(\boldsymbol{\xi}_{i}\right) \otimes \mathcal{Y}_{1}\left(\boldsymbol{\xi}_{i}\right)\right]_{l} \otimes \mathcal{Y}_{1}(\boldsymbol{r})\right]_{1 \mu} \\
& =\frac{1}{3} \xi_{i}^{2} \mathcal{Y}_{1 \mu}(\boldsymbol{r})-\sqrt{\frac{8 \pi}{9}}\left[\mathcal{Y}_{2}\left(\boldsymbol{\xi}_{i}\right) \otimes \mathcal{Y}_{1}(\boldsymbol{r})\right]_{1 \mu} .
\end{aligned}
$$

Substituting Eq. (C6) to Eq. (C2), one obtains Eq. (32).

[1] N. Paar, D. Vretenar, E. Khan, and G. Colò, Rep. Prog. Phys. 70, 691 (2007).

[2] D. Savran, T. Aumann, and A. Zilges, Prog. Part. Nucl. Phys. 70, 210 (2013).

[3] R. Mohan, M. Danos, and L. C. Biedenharn, Phys. Rev. C 3, 1740 (1971).

[4] Y. Suzuki, K. Ikeda, and H. Sato, Prog. Theor. Phys. 83, 180 (1990).

[5] P. Van Isacker, M. A. Nagarajan, and D. D. Warner, Phys. Rev. C 45, R13 (1992).

[6] A. Carbone, G. Colò, A. Bracco, L.-G. Cao, P. F. Bortignon, F. Camera, and O. Wieland, Phys. Rev. C 81, 041301 (2010).

[7] P.-G. Reinhard and W. Nazarewicz, Phys. Rev. C 81, 051303 (2010).

[8] J. Piekarewicz, Phys. Rev. C 83, 034319 (2011).

[9] J. Piekarewicz, B. K. Agrawal, G. Colò, W. Nazarewicz, N. Paar, P.-G. Reinhard, X. Roca-Maza, and D. Vretenar, Phys. Rev. C 85, 041302 (2012).

[10] T. Inakura, T. Nakatsukasa, and K. Yabana, Phys. Rev. C 88, 051305 (2013).

[11] S. Goriely, Phys. Lett. B 436, 10 (1998).

[12] S. Goriely, E. Khan, and M. Samyn, Nucl. Phys. A 739, 331 (2004).

[13] E. Litvinova, H. Loens, K. Langanke, G. Martínez-Pinedo, T. Rauscher, P. Ring, F.-K. Thielemann, and V. Tselyaev, Nucl. Phys. A 823, 26 (2009).
[14] J. Gibelin, D. Beaumel, T. Motobayashi, Y. Blumenfeld, N. Aoi, H. Baba, Z. Elekes, S. Fortier, N. Frascaria, N. Fukuda, T. Gomi, K. Ishikawa, Y. Kondo, T. Kubo, V. Lima, T. Nakamura, A. Saito, Y. Satou, J.-A. Scarpaci, E. Takeshita, S. Takeuchi, T. Teranishi, Y. Togano, A. M. Vinodkumar, Y. Yanagisawa, and K. Yoshida, Phys. Rev. Lett. 101, 212503 (2008).

[15] L.-G. Cao and Z.-Y. Ma, Phys. Rev. C 71, 034305 (2005).

[16] S. Péru, H. Goutte, and J. Berger, Nucl. Phys. A 788, 44 (2007).

[17] K. Yoshida and N. V. Giai, Phys. Rev. C 78, 064316 (2008).

[18] K. Yoshida and N. V. Giai, Phys. Rev. C 78, 014305 (2008).

[19] T. Inakura, T. Nakatsukasa, and K. Yabana, Phys. Rev. C 84, 021302 (2011).

[20] M. Martini, S. Péru, and M. Dupuis, Phys. Rev. C 83, 034309 (2011)

[21] Y. Hashimoto, Eur. Phys. J. A 48, 55 (2012).

[22] S. Ebata, T. Nakatsukasa, and T. Inakura, Phys. Rev. C 90, 024303 (2014).

[23] S. Péru and M. Martini, Eur. Phys. J. A 50, 88 (2014).

[24] B. Löher, D. Savran, T. Aumann, J. Beller, M. Bhike, N. Cooper, V. Derya, M. Duchêne, J. Endres, A. Hennig, P. Humby, J. Isaak, J. H. Kelley, M. Knörzer, N. Pietralla, V. Yu. Ponomarev, C. Romig, M. Scheck, H. Scheit, J. Silva, A. P. Tonchev, W. Tornow, F. Wamers, H. Weller, V. Werner, and A. Zilges, Phys. Lett. B 756, 72 (2016).

[25] O. Sorlin and M.-G. Porquet, Prog. Part. Nucl. Phys. 61, 602 (2008). 
[26] Y. Kanada-En'yo, M. Kimura, and H. Horiuchi, C. R. Phys. 4, 497 (2003).

[27] Y. Kanada-En'yo, M. Kimura, and A. Ono, Prog. Theor. Exp. Phys. 2012, 1A202 (2012).

[28] Y. Kanada-En'yo, Phys. Rev. C 89, 024302 (2014).

[29] Y. Chiba and M. Kimura, Phys. Rev. C 91, 061302 (2015).

[30] Y. Kanada-En'yo, Phys. Rev. C 93, 024322 (2016).

[31] Y. Kanada-En'yo, Phys. Rev. C 93, 054307 (2016).

[32] J. Berger, M. Girod, and D. Gogny, Comput. Phys. Commun. 63, 365 (1991).

[33] M. Kimura, Phys. Rev. C 69, 044319 (2004).

[34] Equation (4) is given by a coherent state form. It is different from Eq. (3) in Ref. [33] by the phase factor $e^{-\frac{1}{2} Z_{i}^{2}}$ and $Z_{i \sigma}$ is scaled by $1 / \sqrt{\nu_{\sigma}}$.

[35] M. Kimura, R. Yoshida, and M. Isaka, Prog. Theor. Phys. 127, 287 (2012).

[36] Y. Kanada-En'yo and M. Kimura, Phys. Rev. C 72, 064301 (2005).

[37] D. L. Hill and J. A. Wheeler, Phys. Rev. 89, 1102 (1953).

[38] J. J. Griffin and J. A. Wheeler, Phys. Rev. 108, 311 (1957).

[39] S. W. Padgett, V. Tripathi, S. L. Tabor, P. F. Mantica, C. R. Hoffman, M. Wiedeking, A. D. Davies, S. N. Liddick, W. F. Mueller, A. Stolz, and B. E. Tomlin, Phys. Rev. C 72, 064330 (2005).

[40] M. Belleguic, F. Azaiez, Z. Dombrádi, M. J. LopezJimenez, T. Otsuka, M. G. Saint-Laurent, D. Sohler, O. Sorlin, M. Stanoiu, Y. Utsuno, Y.-E. Penionzhkevich, N. L. Achouri, J. C. Angelique, C. Borcea, C. Bourgeois, J. M. Daugas, F. D. Oliveira-Santos, Z. Dlouhy, C. Donzaud, J. Duprat, Z. Elekes, S. Grévy, D. Guillemaud Mueller, S. Leenhardt, M. Lewitowicz, S. M. Lukyanov, W. Mittig, M. G. Porquet, F. Pougheon, P. Roussel Chomaz, H. Savajols, Y. Sobolev, C. Stodel, and J. Timár, Phys. Rev. C 72, 054316 (2005).

[41] A. Obertelli, A. Gillibert, N. Alamanos, M. A. G. Alvarez, F. Auger, R. Dayras, A. Drouart, N. Keeley, V. Lapoux, X. Mougeot, L. Nalpas, E. Pollacco, F. Skaza, C. Theisen, G. de France, B. Jurado, W. Mittig, F. Rejmund, M. Rejmund, P. Roussel-Chomaz, H. Savajols, A. Pakou, and N. Patronis, Phys. Rev. C 74, 064305 (2006).

[42] W. N. Catford, C. N. Timis, R. C. Lemmon, M. Labiche, N. A. Orr, B. Fernández-Domínguez, R. Chapman, M. Freer, M. Chartier, H. Savajols, M. Rejmund, N. L. Achouri, N. Amzal, N. I. Ashwood, T. D. Baldwin, M. Burns, L. Caballero, J. M. Casadjian, N. Curtis, G. de France, W. Gelletly, X. Liang, S. D. Pain, V.P. E. Pucknell, B. Rubio, O. Sorlin, K. Spohr, C. Theisen, and D. D. Warner, Phys. Rev. Lett. 104, 192501 (2010).

[43] R. Rodríguez-Guzmán, J. Egido, and L. Robledo, Eur. Phys. J. A 17, 37 (2003).

[44] A. Obertelli, S. Péru, J. P. Delaroche, A. Gillibert, M. Girod, and H. Goutte, Phys. Rev. C 71, 024304 (2005).

[45] S. Raman, C. Malarkey, W. Milner, C. Nestor, and P. Stelson, At. Data Nucl. Data Tables 36, 1 (1987).

[46] J. Gibelin, D. Beaumel, T. Motobayashi, N. Aoi, H. Baba, Y. Blumenfeld, Z. Dombrádi, Z. Elekes, S. Fortier, N. Frascaria, N. Fukuda, T. Gomi, K. Ishikawa, Y. Kondo, T. Kubo, V. Lima, T. Nakamura, A. Saito, Y. Satou, E. Takeshita, S. Takeuchi, T. Teranishi, Y. Togano, A. M. Vinodkumar, Y. Yanagisawa, and K. Yoshida, Phys. Rev. C 75, 057306 (2007).

[47] J. R. Terry, D. Bazin, B. A. Brown, C. M. Campbell, J. A. Church, J. M. Cook, A. D. Davies, D.-C. Dinca, J. Enders, A. Gade, T. Glasmacher, P. G. Hansen, J. L. Lecouey, T. Otsukad,
B. Pritychenko, B. M. Sherrill, J. A. Tostevin, Y. Utsuno, K. Yoneda, and H. Zwahlen, Phys. Lett. B 640, 86 (2006).

[48] A. T. Reed, O. Tarasov, R. D. Page, D. Guillemaud-Mueller, Y. E. Penionzhkevich, R. G. Allatt, J. C. Angélique, R. Anne, C. Borcea, V. Burjan, W. N. Catford, Z. Dlouhý, C. Donzaud, S. Grévy, M. Lewitowicz, S. M. Lukyanov, F. M. Marqués, G. Martinez, A. C. Mueller, P. J. Nolan, J. Novák, N. A. Orr, F. Pougheon, P. H. Regan, M. G. Saint-Laurent, T. Siiskonen, O. Sokol, O. Sorlin, J. Suhonen, W. Trinder, and S. M. Vincent, Phys. Rev. C 60, 024311 (1999).

[49] J. Terry and J. Lecouey, Nucl. Phys. A 734, 469 (2004).

[50] W. N. Catford, R. C. Lemmon, M. Labiche, C. N. Timis, N. A. Orr, L. Caballero, R. Chapman, M. Chartier, M. Rejmund, and H. Savajols, Eur. Phys. J. A - Hadron. Nucl. 25, 245 (2005).

[51] T. Nakamura, N. Kobayashi, Y. Kondo, Y. Satou, N. Aoi, H. Baba, S. Deguchi, N. Fukuda, J. Gibelin, N. Inabe, M. Ishihara, D. Kameda, Y. Kawada, T. Kubo, K. Kusaka, A. Mengoni, T. Motobayashi, T. Ohnishi, M. Ohtake, N. A. Orr, H. Otsu, T. Otsuka, A. Saito, H. Sakurai, S. Shimoura, T. Sumikama, H. Takeda, E. Takeshita, M. Takechi, S. Takeuchi, K. Tanaka, K. N. Tanaka, N. Tanaka, Y. Togano, Y. Utsuno, K. Yoneda, A. Yoshida, and K. Yoshida, Phys. Rev. Lett. 103, 262501 (2009).

[52] I. Hamamoto, Phys. Rev. C 81, 021304 (2010).

[53] W. Horiuchi, Y. Suzuki, P. Capel, and D. Baye, Phys. Rev. C 81, 024606 (2010).

[54] K. Minomo, T. Sumi, M. Kimura, K. Ogata, Y. R. Shimizu, and M. Yahiro, Phys. Rev. Lett. 108, 052503 (2012).

[55] M. Takechi, T. Ohtsubo, M. Fukuda, D. Nishimura, T. Kuboki, T. Suzuki, T. Yamaguchi, A. Ozawa, T. Moriguchi, H. Ooishi, D. Nagae, H. Suzuki, S. Suzuki, T. Izumikawa, T. Sumikama, M. Ishihara, H. Geissel, N. Aoi, R.-J. Chen, D.-Q. Fang, N. Fukuda, I. Hachiuma, N. Inabe, Y. Ishibashi, Y. Ito, D. Kameda, T. Kubo, K. Kusaka, M. Lantz, Y.-G. Ma, K. Matsuta, M. Mihara, Y. Miyashita, S. Momota, K. Namihira, M. Nagashima, Y. Ohkuma, T. Ohnishi, M. Ohtake, K. Ogawa, H. Sakurai, Y. Shimbara, T. Suda, H. Takeda, S. Takeuchi, K. Tanaka, R. Watanabe, M. Winkler, Y. Yanagisawa, Y. Yasuda, K. Yoshinaga, A. Yoshida, and K. Yoshida, Phys. Lett. B 707, 357 (2012).

[56] K. Yoshida, Phys. Rev. C 80, 044324 (2009).

[57] M. Martini, S. Péru, S. Hilaire, S. Goriely, and F. Lechaftois, Phys. Rev. C 94, 014304 (2016).

[58] Y. Chiba, M. Kimura, and Y. Taniguchi, Phys. Rev. C 93, 034319 (2016)

[59] However, it must be noted that this expectation is invalid for the halo nuclei in which the valence neutron has a huge radius. For halo nuclei, the contribution from the second term will predominate over other terms, and the halo nuclei should have a strong IS dipole mode at small excitation energy. Indeed, this was already pointed out for ${ }^{6} \mathrm{He}[65]$ theoretically, and recently observed in ${ }^{11} \mathrm{Li}[66]$.

[60] N. Paar, Y. F. Niu, D. Vretenar, and J. Meng, Phys. Rev. Lett. 103, 032502 (2009).

[61] D. Vretenar, Y. F. Niu, N. Paar, and J. Meng, Phys. Rev. C 85, 044317 (2012).

[62] X. Roca-Maza, G. Pozzi, M. Brenna, K. Mizuyama, and G. Colò, Phys. Rev. C 85, 024601 (2012).

[63] H. Nakada, T. Inakura, and H. Sawai, Phys. Rev. C 87, 034302 (2013).

[64] F. Kobayashi and Y. Kanada-En’yo, Phys. Rev. C 93, 024310 (2016). 
[65] D. Mikami, W. Horiuchi, and Y. Suzuki, Phys. Rev. C 89, 064303 (2014).

[66] R. Kanungo, A. Sanetullaev, J. Tanaka, S. Ishimoto, G. Hagen, T. Myo, T. Suzuki, C. Andreoiu, P. Bender, A. A. Chen, B. Davids, J. Fallis, J. P. Fortin, N. Galinski, A. T. Gallant, P. E. Garrett,
G. Hackman, B. Hadinia, G. Jansen, M. Keefe, R. Krücken, J. Lighthall, E. McNeice, D. Miller, T. Otsuka, J. Purcell, J. S. Randhawa, T. Roger, A. Rojas, H. Savajols, A. Shotter, I. Tanihata, I. J. Thompson, C. Unsworth, P. Voss, and Z. Wang, Phys. Rev. Lett. 114, 192502 (2015). 